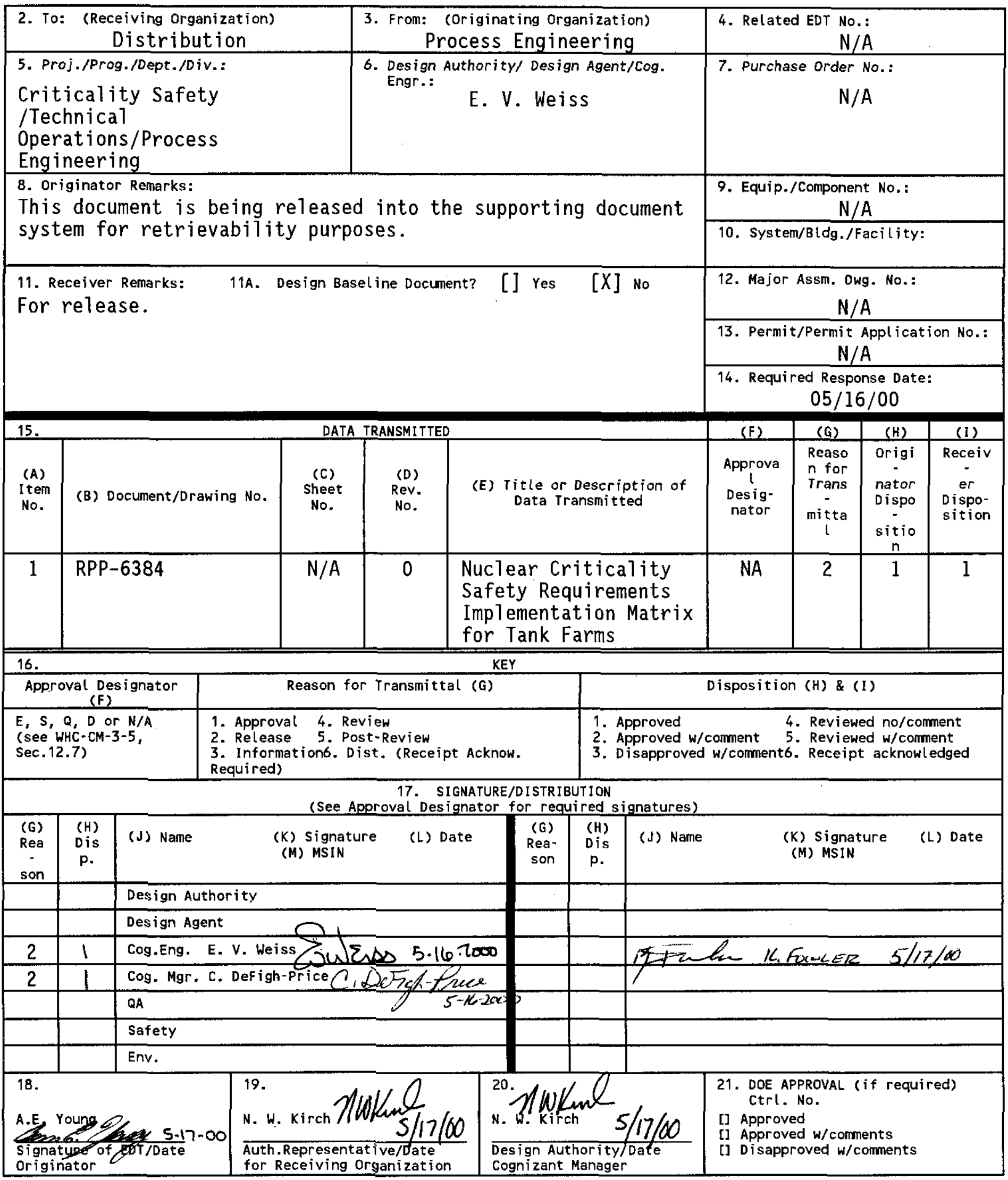

BD-7400-172-2(05/96) GEF097 


\section{Nuclear Criticality Safety Requirements Implementation Matrix for Tank Farms}

\section{E. E. Weiss}

CH2M HILL Hanford Group, Inc., Rich1 and, WA 99352

U.S. Department of Energy Contract DE-AC06-96RL13200

EDT/ECN: EDT-628378

Org Code: 74B50

B\&R Code: EW 3120074
UC: 2070

CACN/COA: $101965 / A 020$

Total Pages: 50

Key Words: Criticality Safety Requirements, CSR

Abstract: This document provides a detailed matrix of specific Tank Farms nuclear criticality safety program elements indexed to primary requirements documents. These requirements are collected at a highter level in HNF-SD-MP-SRID-001, "Tank Waste Remediation System Standards/Requirements Identification Document." The intended use of this document is to provide a roadmap for implementing procedures and as sessments.

TRADEMARX DISCLAIMER. Reference herein to any specific commercial product, process, or service by trade name, trademark, manufacturer, or otherwise, does not necessarily constitute or imply its endorsement, recommendation, or favoring by the United States Government or any agency thereof or its contractors or subcontractors.

Printed in the United States of America. To obtain copies of this document, contact: Document Control Services, P.O. Box 950, Mailstop H6-08, Richland WA 99352, Phone (509) 372-2420;

Fax (509) 376-4989.
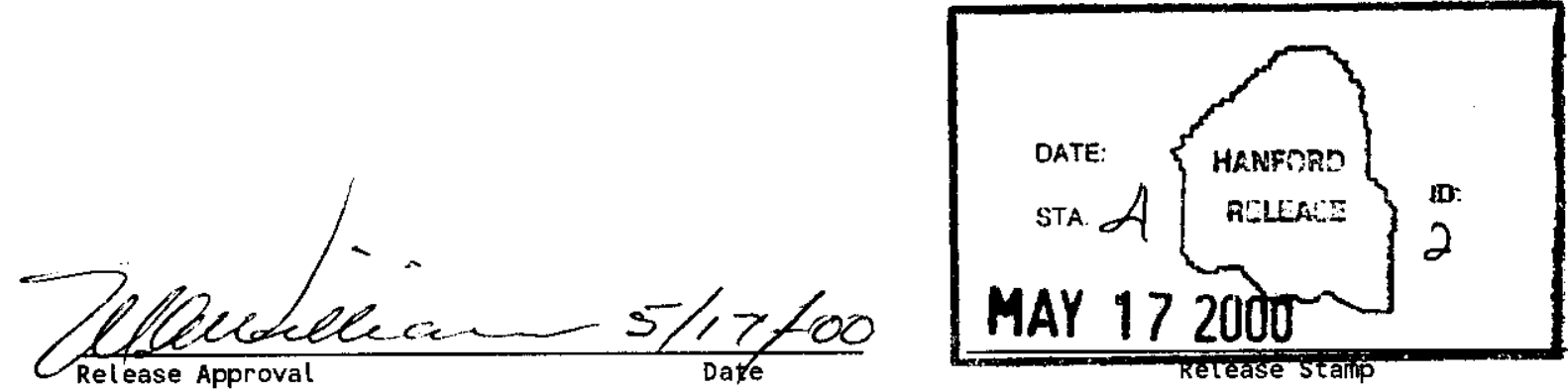

\section{Approved for Public Release}




\section{NUCLEAR CRITICALITY SAFETY REQUIREMENTS IMPLEMENTATION MATRIX FOR TANK FARMS}

Evelyn V. Weiss

May 2000 


\section{EXECUTIVE SUMMARY}

This document provides a detailed matrix of specific Tank Farms nuclear criticality safety program elements indexed to primary requirements documents. These requirements are collected at a higher level in HNF-SD-MP-SRID-001, Tank Waste Remediation System Standards/Requirements Identification Document. The intended use of this document is to provide a roadmap for implementing procedures and assessments. 
This page intentionally left blank 


\section{TABLE OF CONTENTS}

\section{EXECUTIVE SUMMARY}

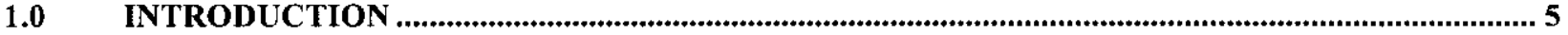

1.1 PURPOSE

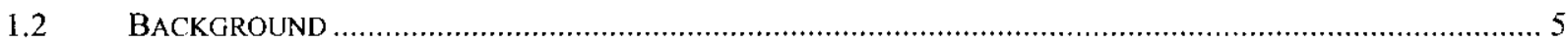

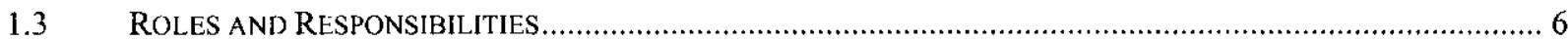

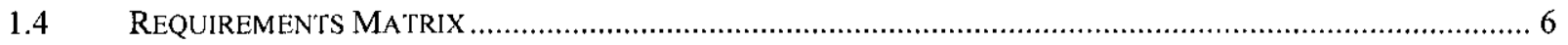

2.0 REQUIREMENTS MATRIX

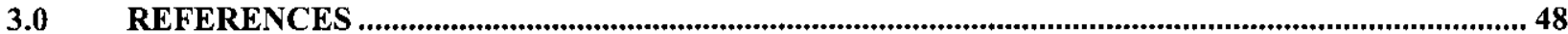


This page intentionally left blank 


\subsection{INTRODUCTION}

\subsection{Purpose}

This document provides a detailed matrix of specific Tank Farms nuclear criticality safety program elements indexed to primary requirements documents. These requirements are collected at a higher level in HNF-SD-MP-SRID-001, Tank Waste Remediation System

Standards/Requirements Identification Document (S/RID). The intended use of this document is to provide a roadmap for implementing procedures and assessments.

The matrix itemizes S/RID compliance items directly related to nuclear criticality safety. Although the primary Department of Energy (DOE) directive related to nuclear criticality safety (DOE 5480.24, "Nuclear Criticality Safety") has been superceded by topical inclusion in DOE 420.1 "Facility Safety," the approved S/RID includes compliance with DOE 5480.24 along with requirements of American National Standards Institute/American Nuclear Society (ANSI/ANS) Series 8 standards of the revision referenced in DOE 5480.24.

The matrix in this document is an update of a tables found in TWR-3721, Tank Waste Remediation System Nuclear Criticality Safety Program Management Review, Appendix B, and document 9454500, "Tank Farms Compliance Assessment and Implementation Plan for DOE Order 5480.24 Nuclear Criticality Safety," (referenced in Internal Memo, E. J. Lipke to T. C. Geer, "Criticality Compliance," September 3, 1997, DE\&S Hanford). The update provides current implementing references and removes safety analysis requirements for which compliance is provided in other Tank Farms programs.

\subsection{Background}

CH2MHill Hanford Group, Inc. (CHG) manages the Hanford Tank Farms portion of the River Protection Project for the DOE Office of River Protection. The Tank Farms nuclear criticality safety program applies to activities associated with the safe receipt, transfer and storage of waste in Tank Farms facilities. Specifically, the program applies to the following fissionable materials storage areas:

- High-level waste (HLW) storage tanks and ancillary equipment,

- Double-contained receiver tanks (DCRTs) and ancillary equipment,

- Miscellaneous inactive storage facilities (MISFs) and other inactive facilities,

- Ponds, cribs, and ditches.

The current Tank Farms mission is to continue to safely store remaining tank waste and to conduct waste retrieval and disposal activities in support of the long-term goal for the complete removal and final disposition of the tank waste. Activities are performed at Tank Farms in a manner that complies with environmental regulations and minimizes public and worker risk.

Collectively, the DSTs and SSTs contain an estimated 500 to $1,000 \mathrm{~kg}(1,100$ to $2,200 \mathrm{lb})$ of plutonium. Analyses of tank waste samples show that the plutonium content of the waste is 
primarily associated with the sludge phase. WHC-SD-WM-TI-725, Tank Farm Nuclear Criticality Review, established that fissionable material in the waste tanks is distributed at subcritical concentrations with excess neutron absorbers present, and that no mechanisms exist to concentrate the fissile material to result in an accidental nuclear criticality. Related accident analyses are documented in HNF-SD-WM-SAR-067, Tank Waste Remediation System Final Safety Analysis Report (FSAR). Section 6.3.2 of the FSAR states that a criticality accident in the Tank Farms is not credible for operations discussed in that document, and that the facility is classified for the purposes of nuclear criticality safety as a Limited Control Facility. The Tank Farms criticality safety program, therefore, consists primarily of administrative controls designed to protect against altering the waste inventory in a way that could increase the probability of a criticality accident. Controls for the program are discussed in HNF-SD-WM-TSR-006, Tank Waste Remediation System Technical Safety Requirements, Administrative Control 5.7, "Nuclear Criticality Safety." The program is described in HNF-IP-1266, Tank Farms Operations Administrative Controls, Chapter 5.7, "Nuclear Criticality Safety."

\subsection{Roles and Responsibilities}

The criticality safety program at Tank Farms is focused on maintaining the facility status as a Limited Control Facility. Organizationally, the program manager and Criticality Safety Representative (CSR) report to the facility manager from within the engineering function. The functional organization chart is shown in Figure 1 below.

\subsection{Requirements Matrix}

The detailed matrix of requirements versus implementing documents is shown in Table 1 later in this document. The table is organized to show:

- Item - a sequential number for ease of reference

- Requirements Identification -- where each individual requirement is captured in either the Tank Farms S/RID document or the ANSI/ANS Standard derived from a DOE directive. In a few cases toward the end of the table, this column is used to cite an S/RID paragraph.

- Requirement Source -- in general a paragraph within a DOE directive, in a few cases shows the Tank Farms source document for a derivative requirements (for example, a Technical Safety Requirement (TSR) which refers back to the FSAR).

- Requirement Text $-\mathbf{a}$ brief quote from the requirements document

- RPP Implementing Document - the document number, and in most cases, the section in which each requirement is implemented.

- Comment - additional text describing the way in which Tank Farms complies with a requirement or a reason why the requirement is not applicable. 
Figure 1. Tank Farms Nuclear Criticality Safety Program

Functional Organization

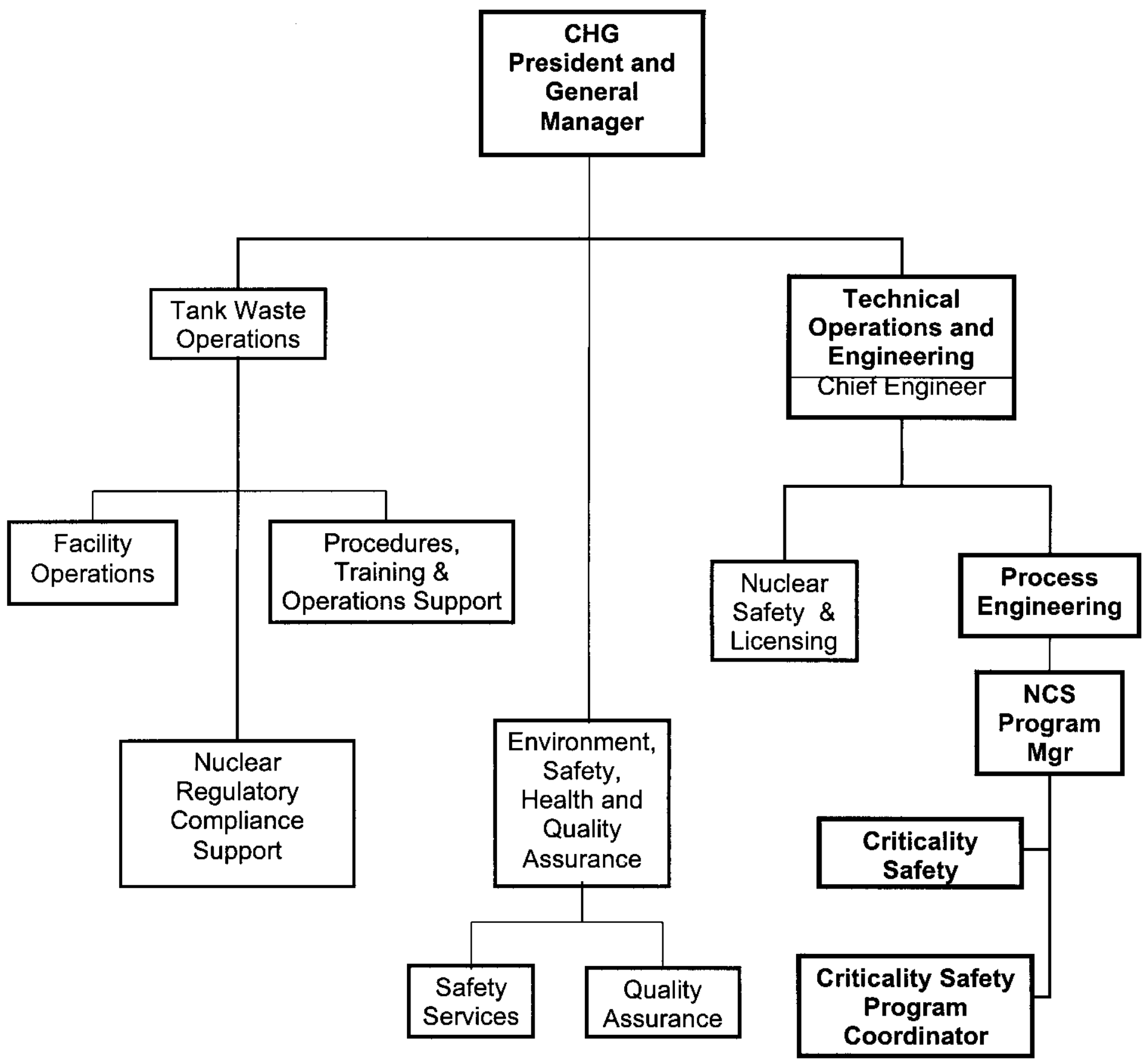

Update: $4 / 24 / 2000$ 


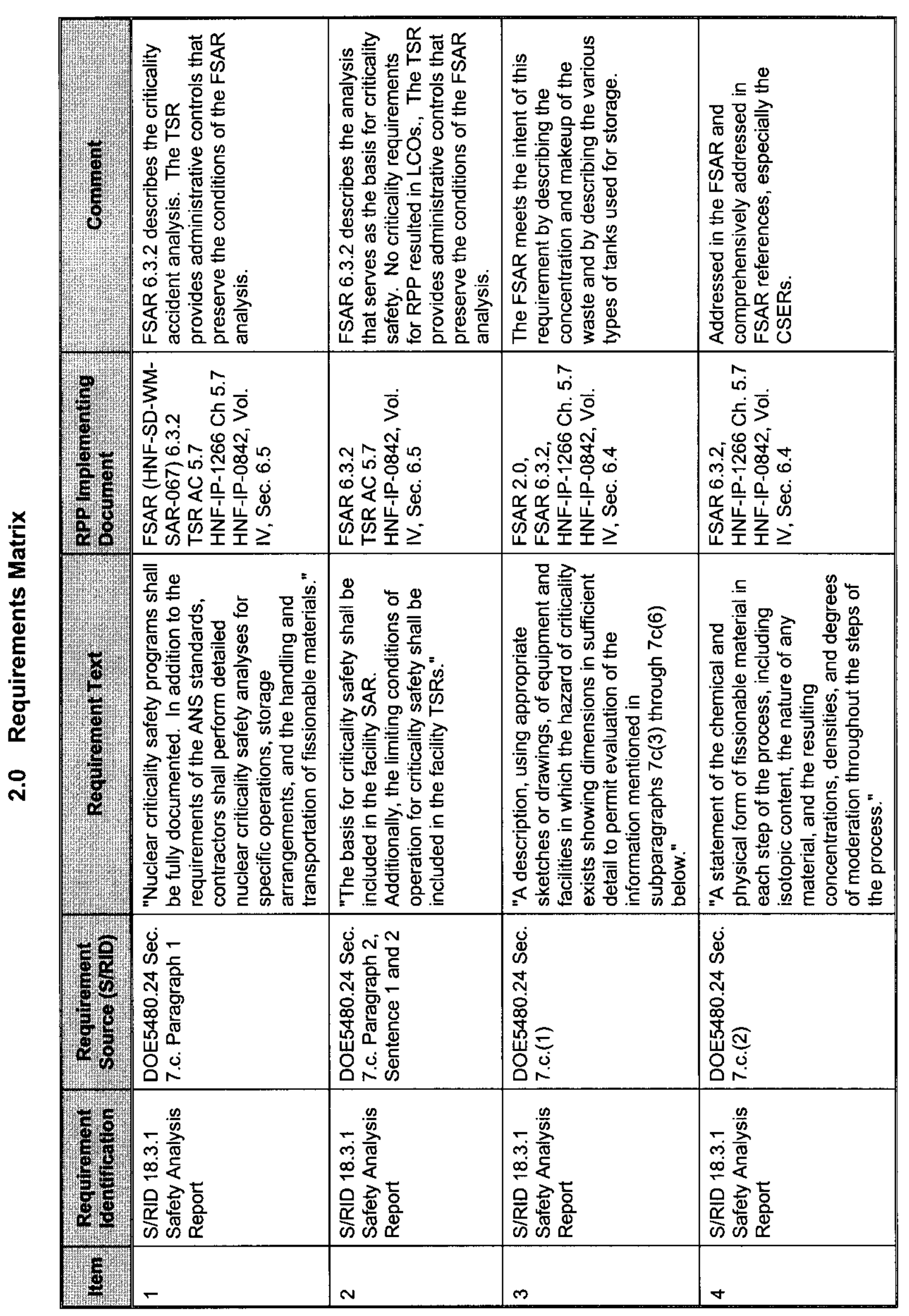




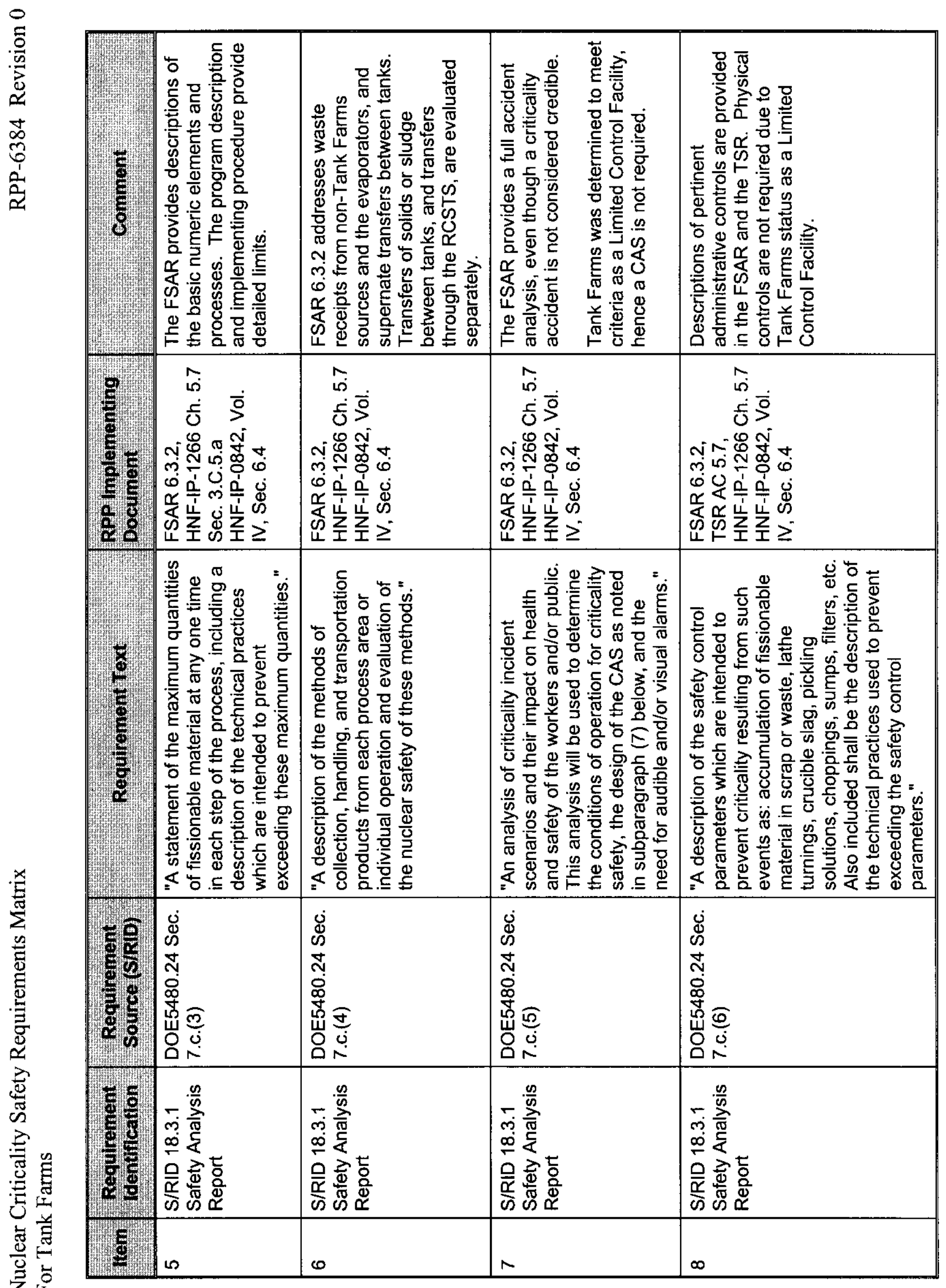




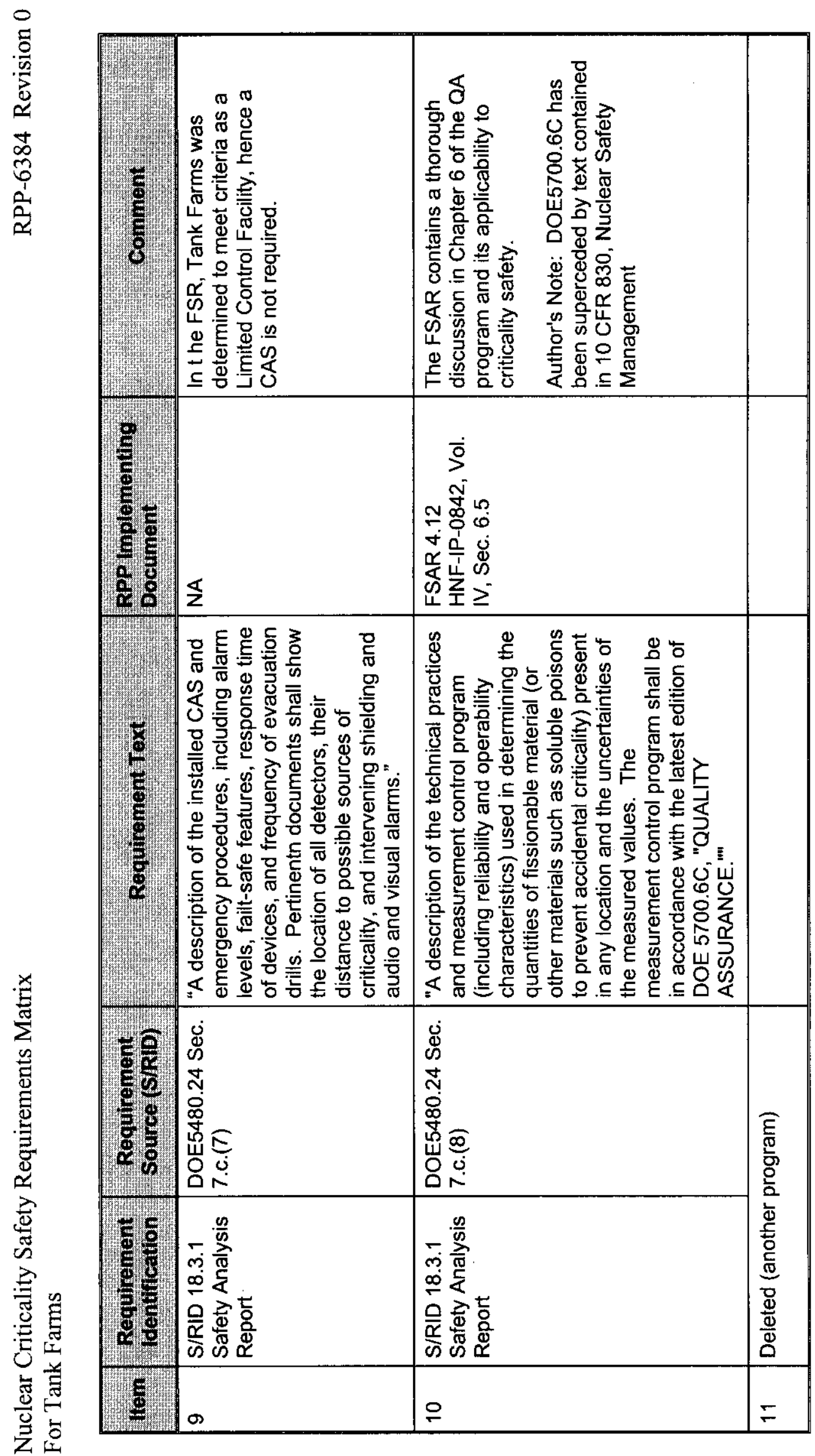




\begin{tabular}{|c|c|c|c|c|}
\hline 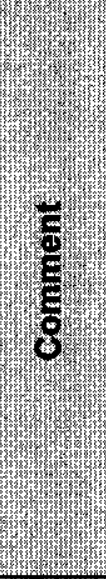 & 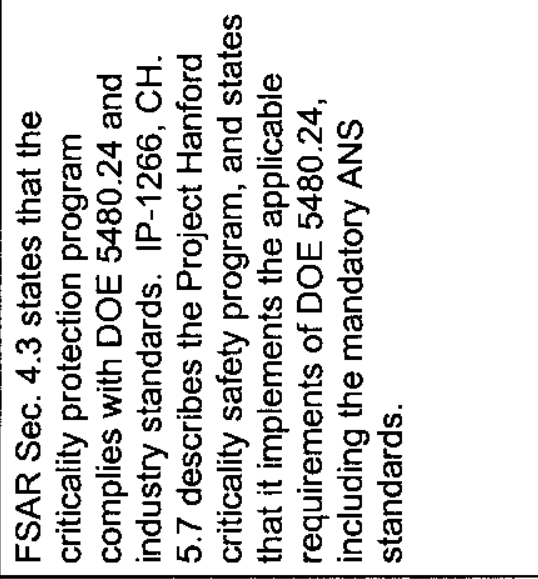 & 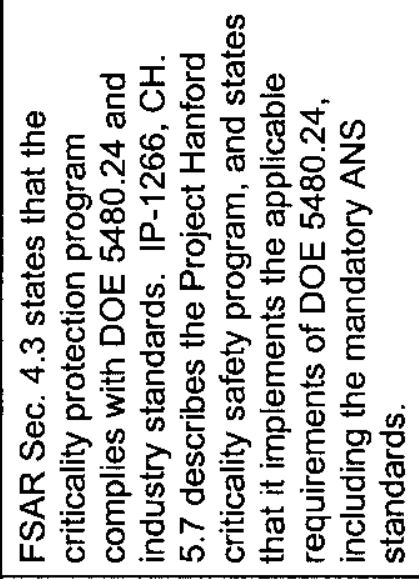 & & \\
\hline 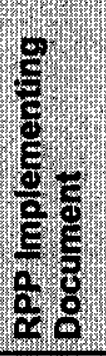 & 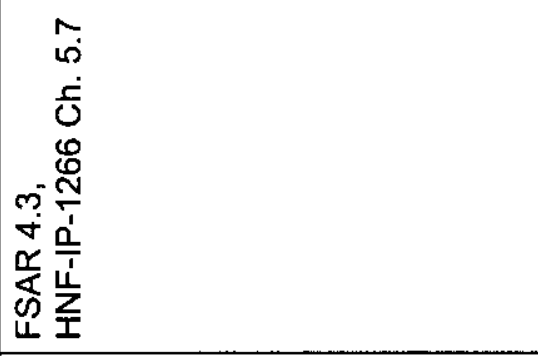 & 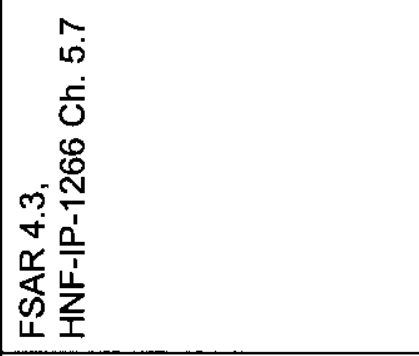 & 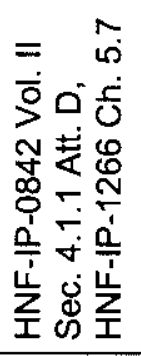 & 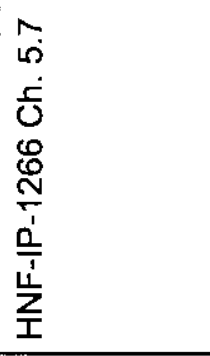 \\
\hline$\frac{8}{8}$ & 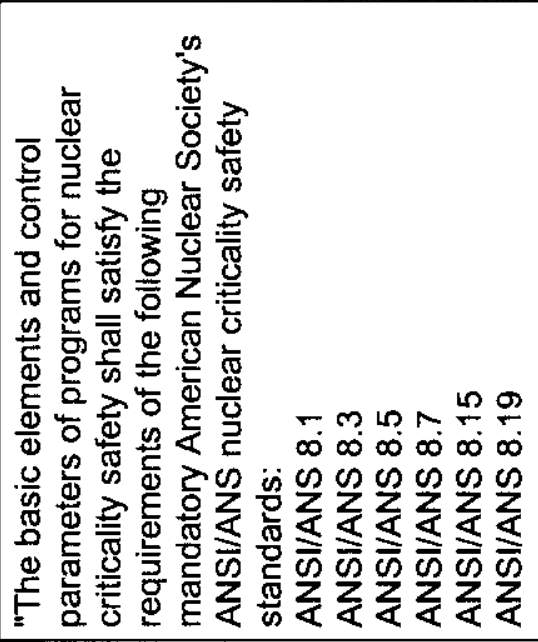 & 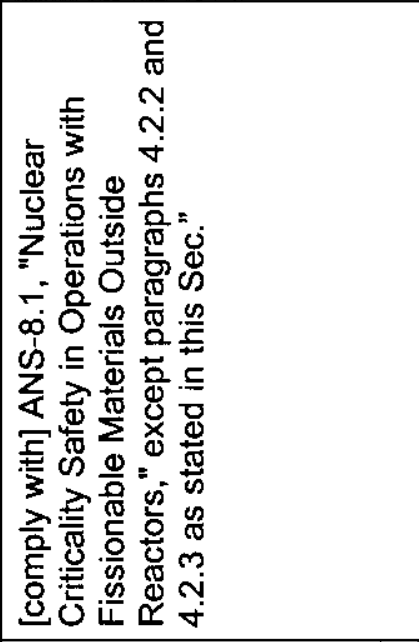 & 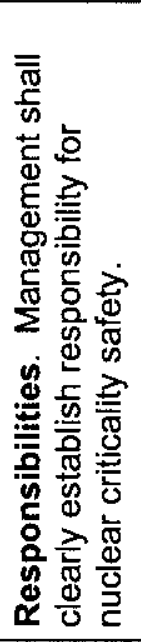 & 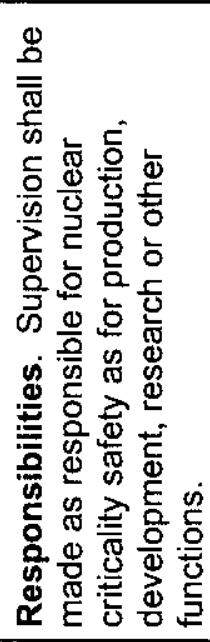 \\
\hline 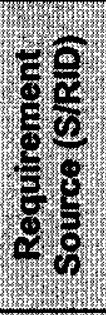 & 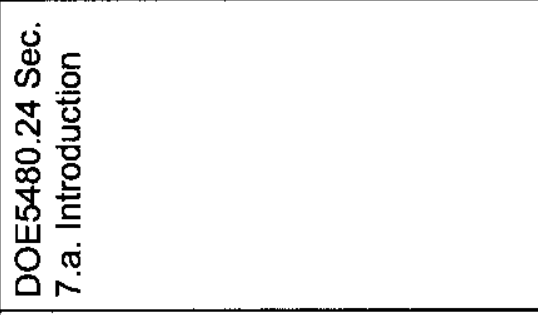 & 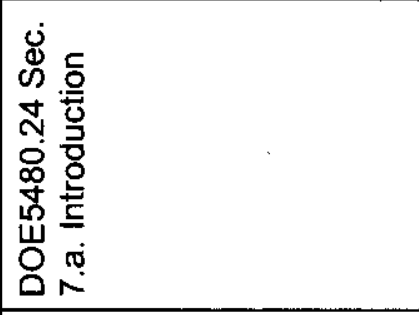 & 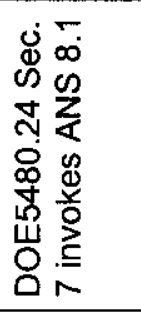 & 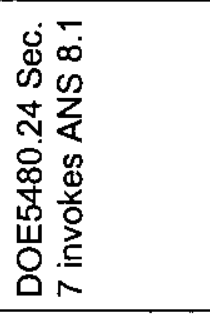 \\
\hline 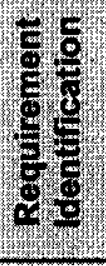 & 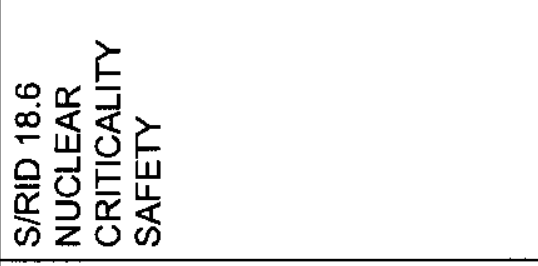 & 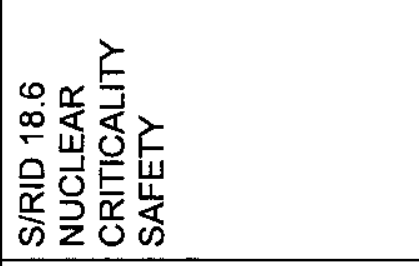 & 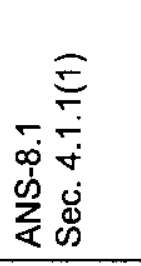 & 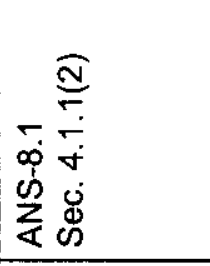 \\
\hline$\frac{9}{9}$ & $\underset{\sim}{\sim}$ & $\stackrel{m}{\sim}$ & $\underset{7}{ }$ & $\stackrel{L}{\sim}$ \\
\hline
\end{tabular}




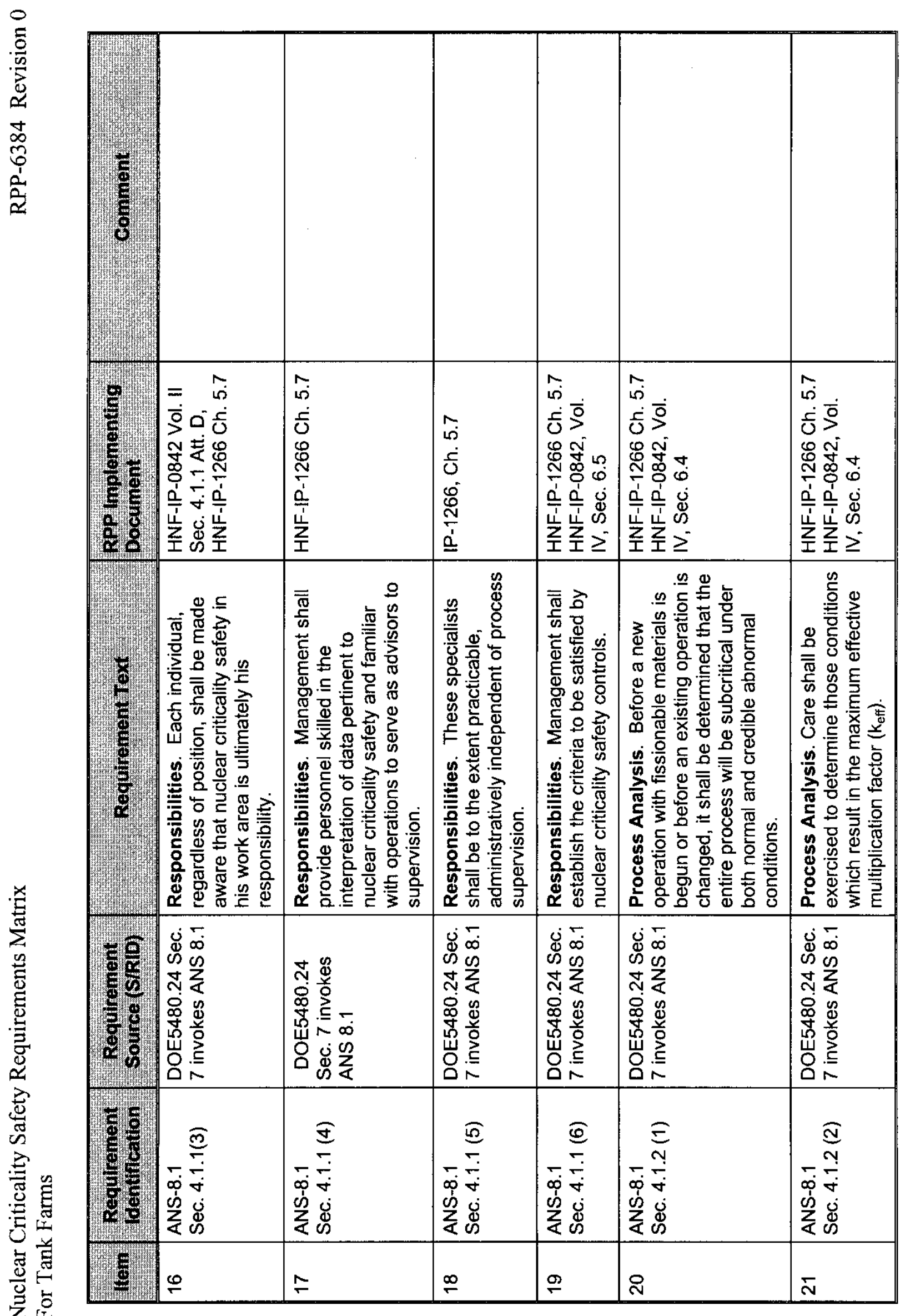




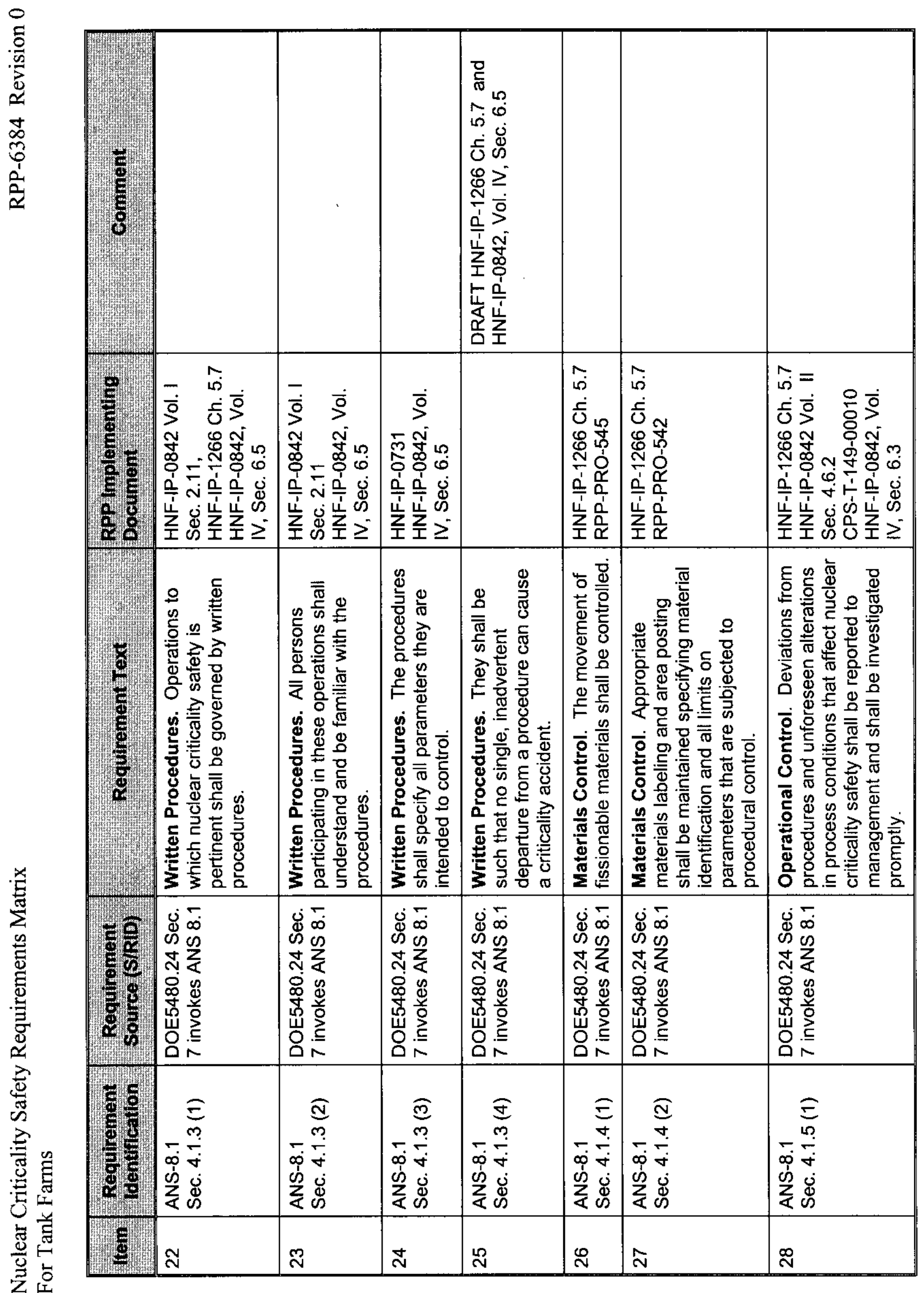




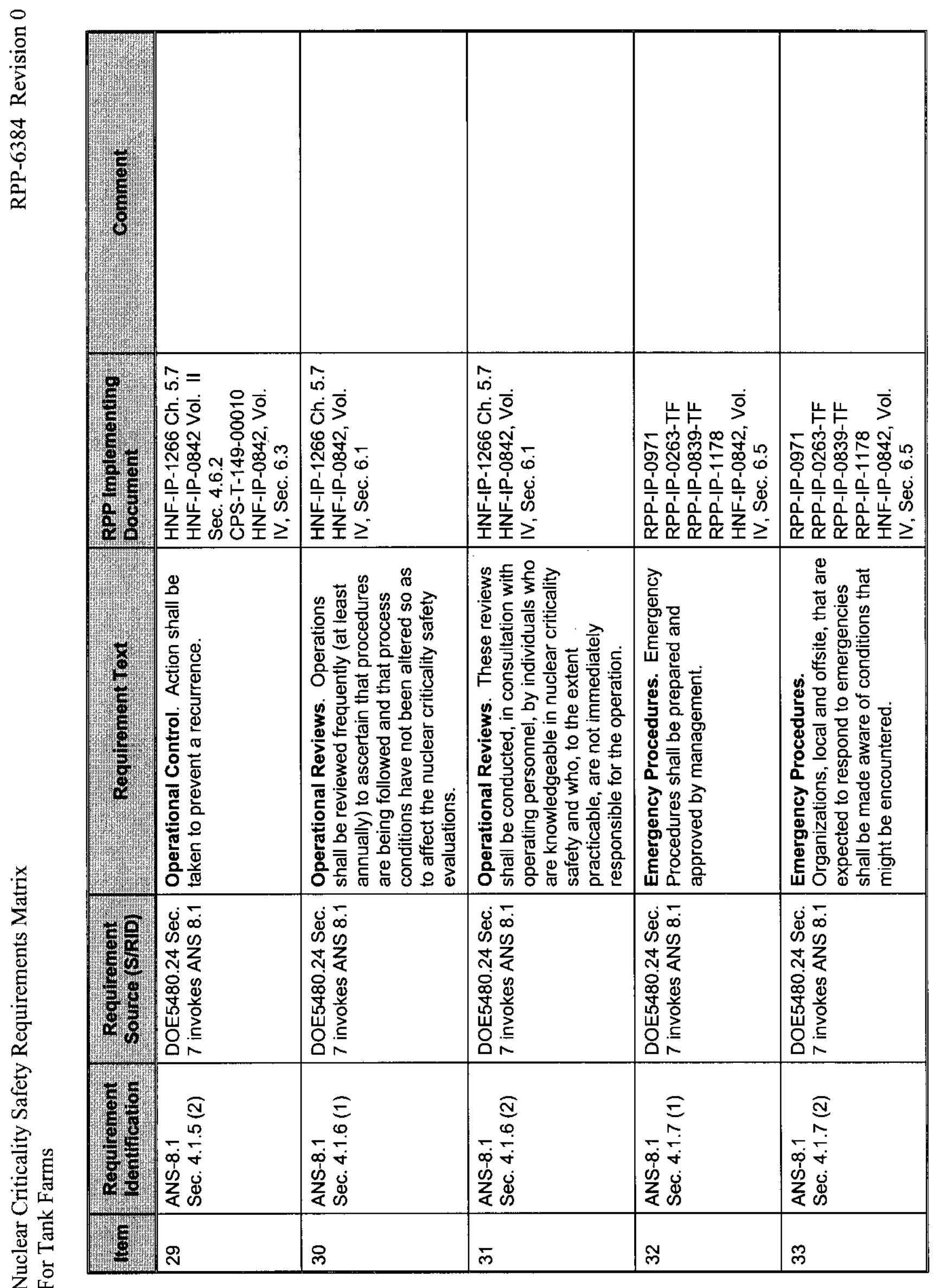




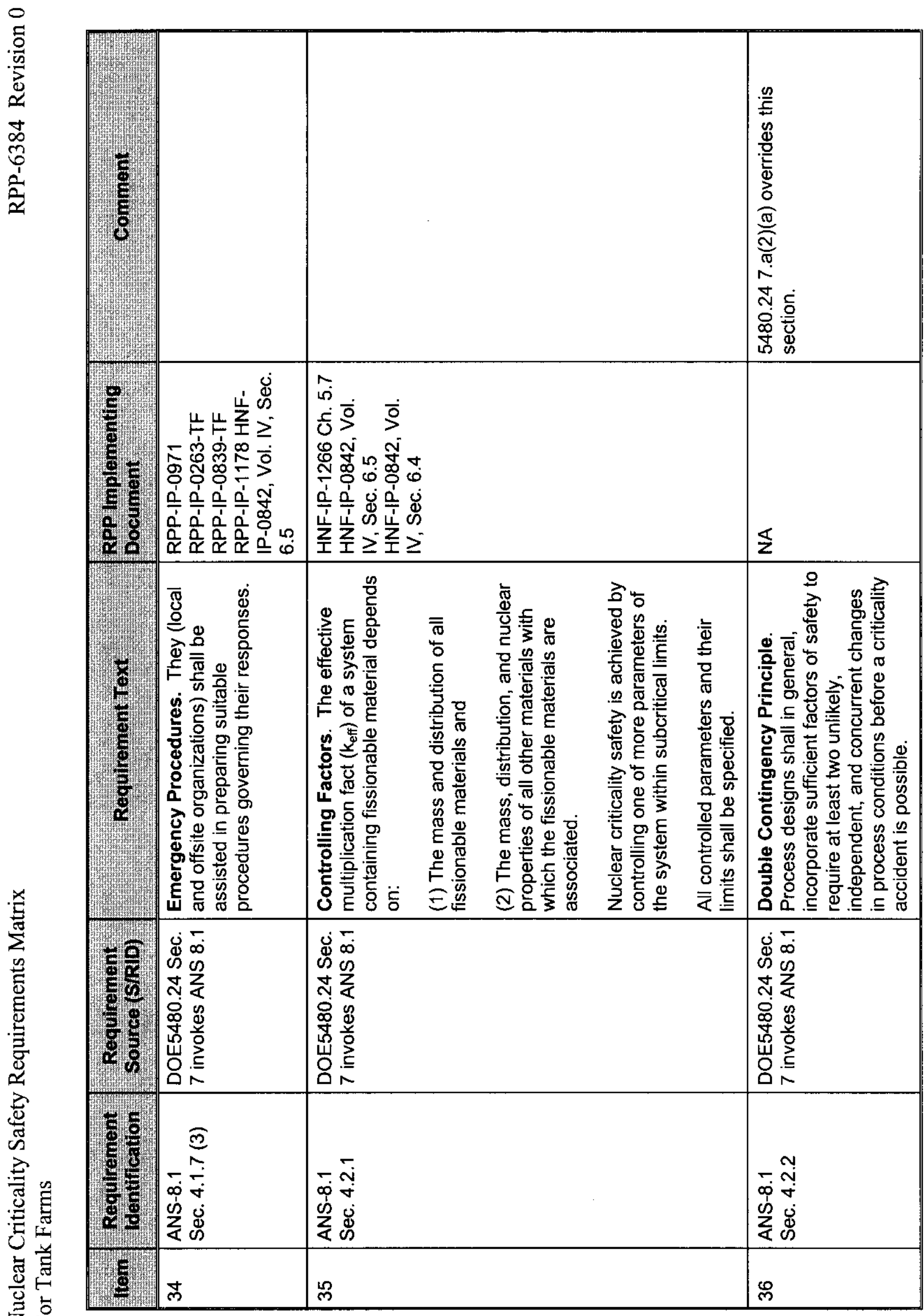




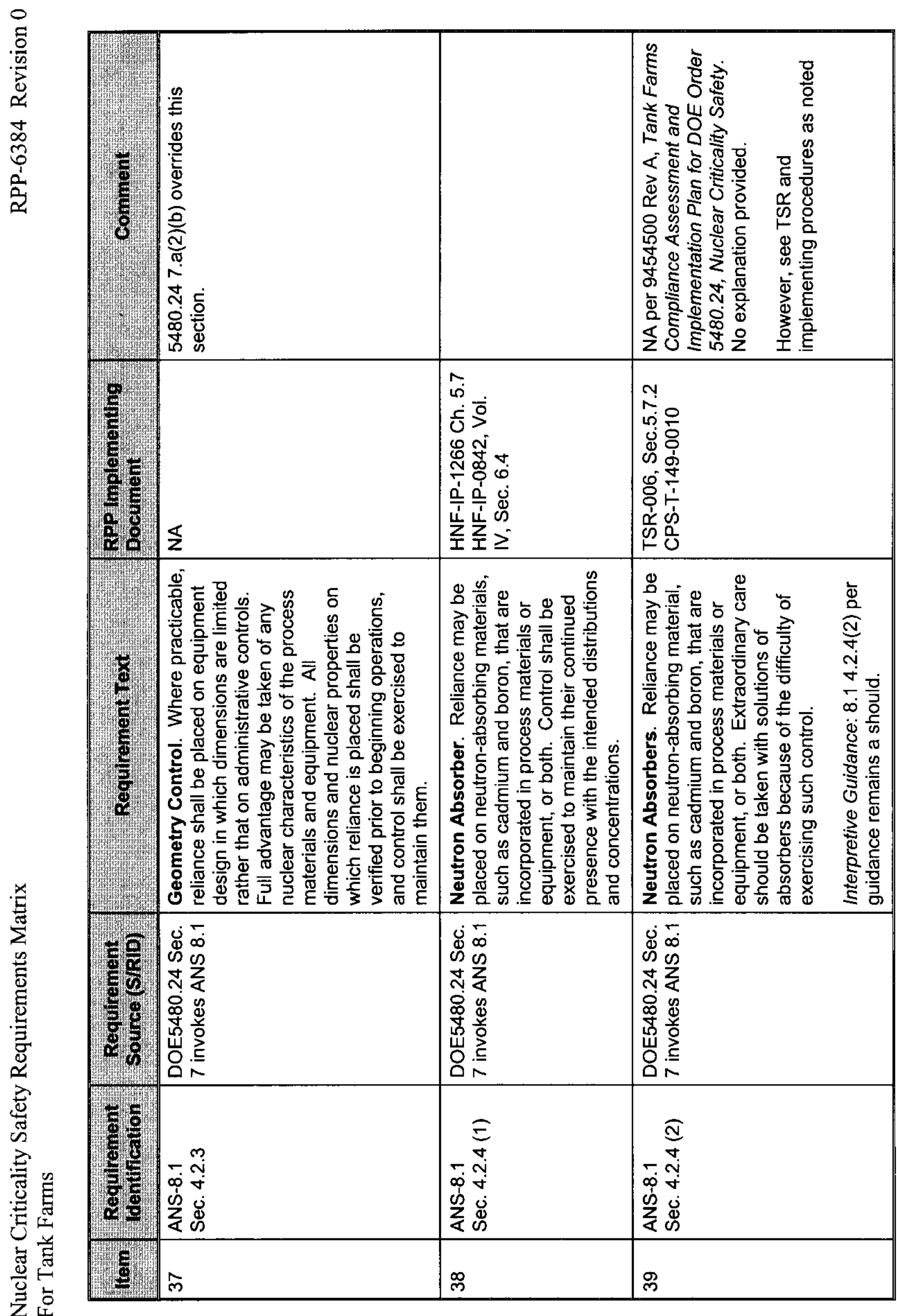




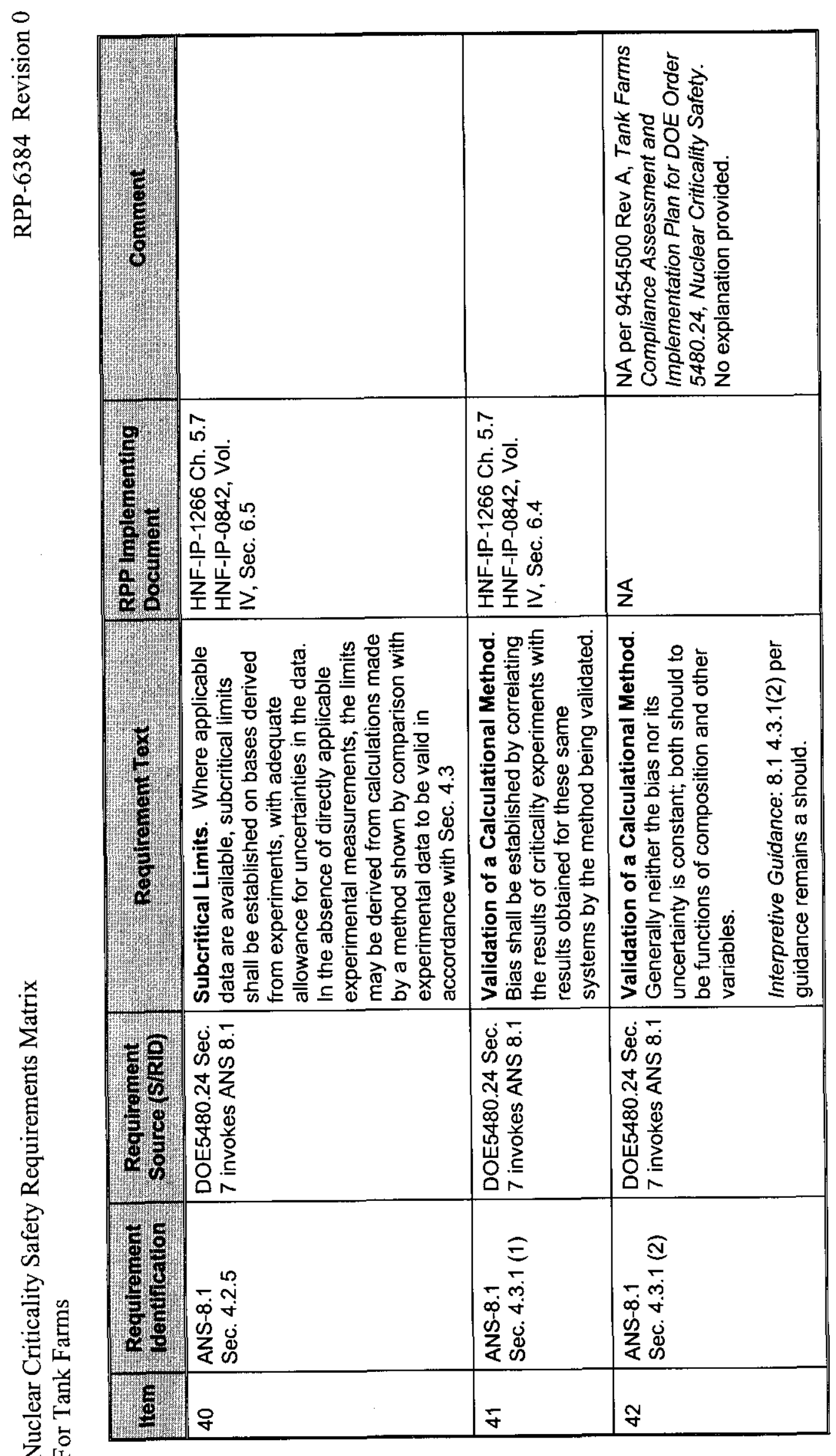




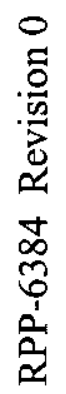

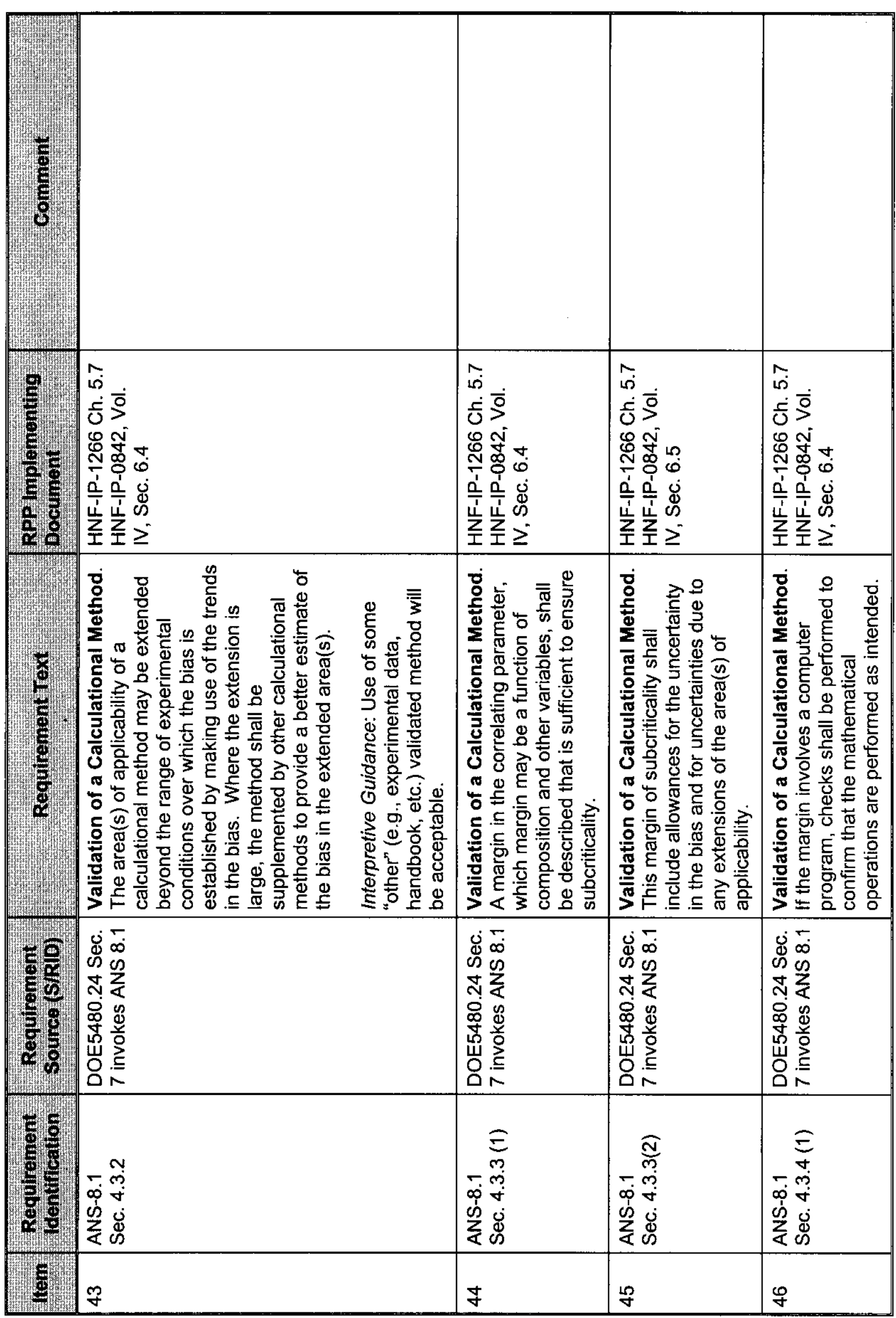




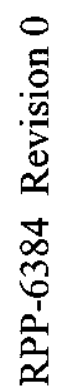

\begin{tabular}{|c|c|c|c|c|c|c|c|c|c|}
\hline 4 & & \multicolumn{2}{|c|}{ 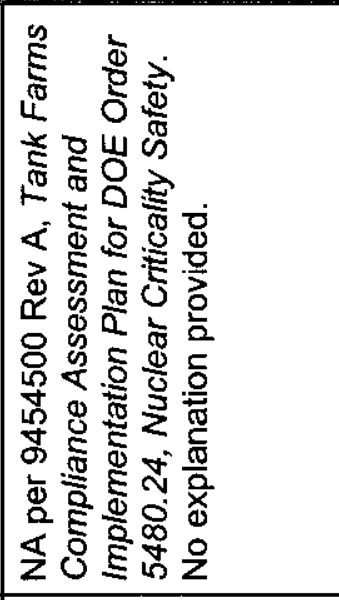 } & & & & & & \\
\hline 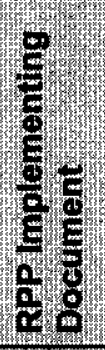 & 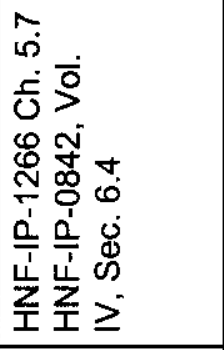 & \multicolumn{2}{|l|}{$\Sigma$} & \multicolumn{4}{|c|}{ 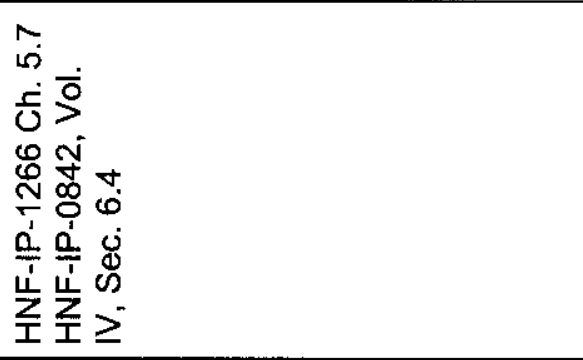 } & \multirow[b]{2}{*}{ 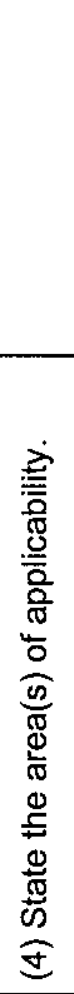 } & \multirow[b]{2}{*}{ 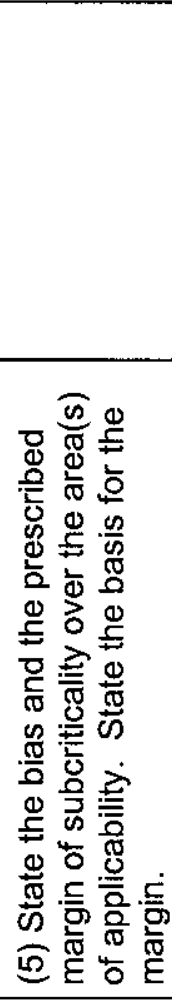 } \\
\hline 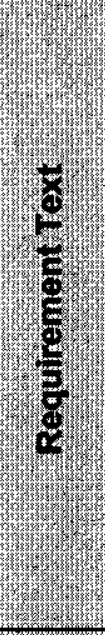 & 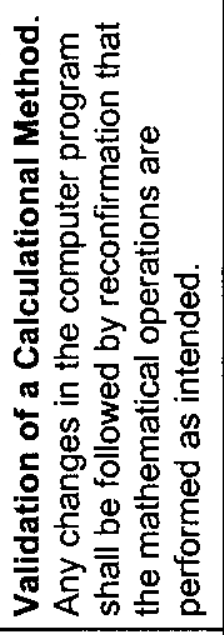 & 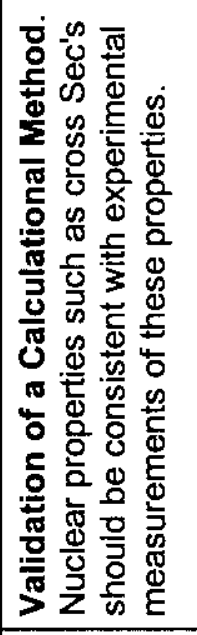 & 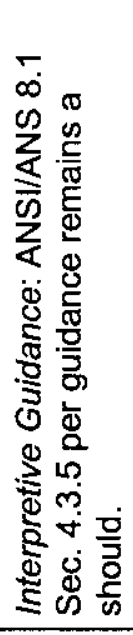 & 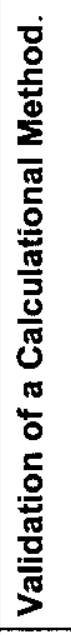 & 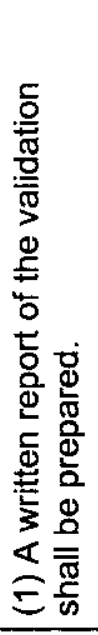 & 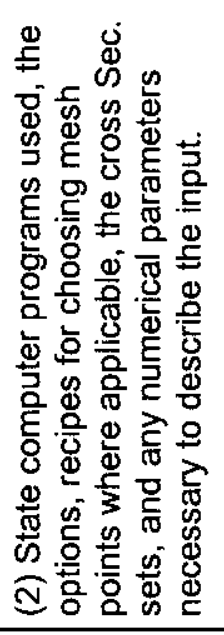 & 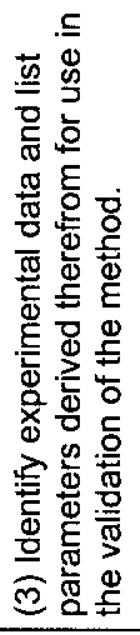 & & \\
\hline 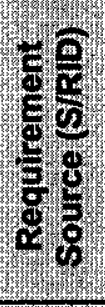 & 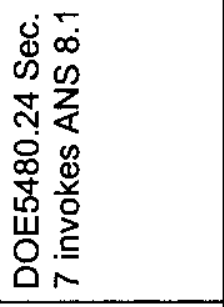 & 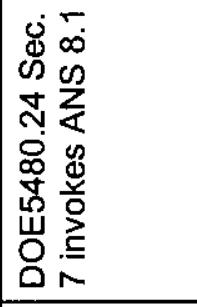 & & 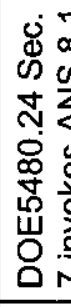 & & & & & \\
\hline 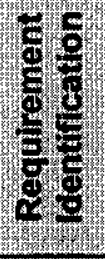 & 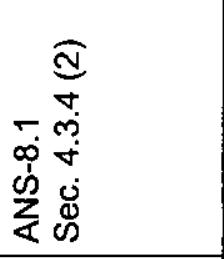 & 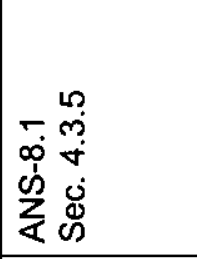 & & 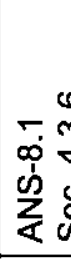 & & & & & \\
\hline E & F & $\stackrel{\infty}{+}$ & & g & & & & & \\
\hline
\end{tabular}




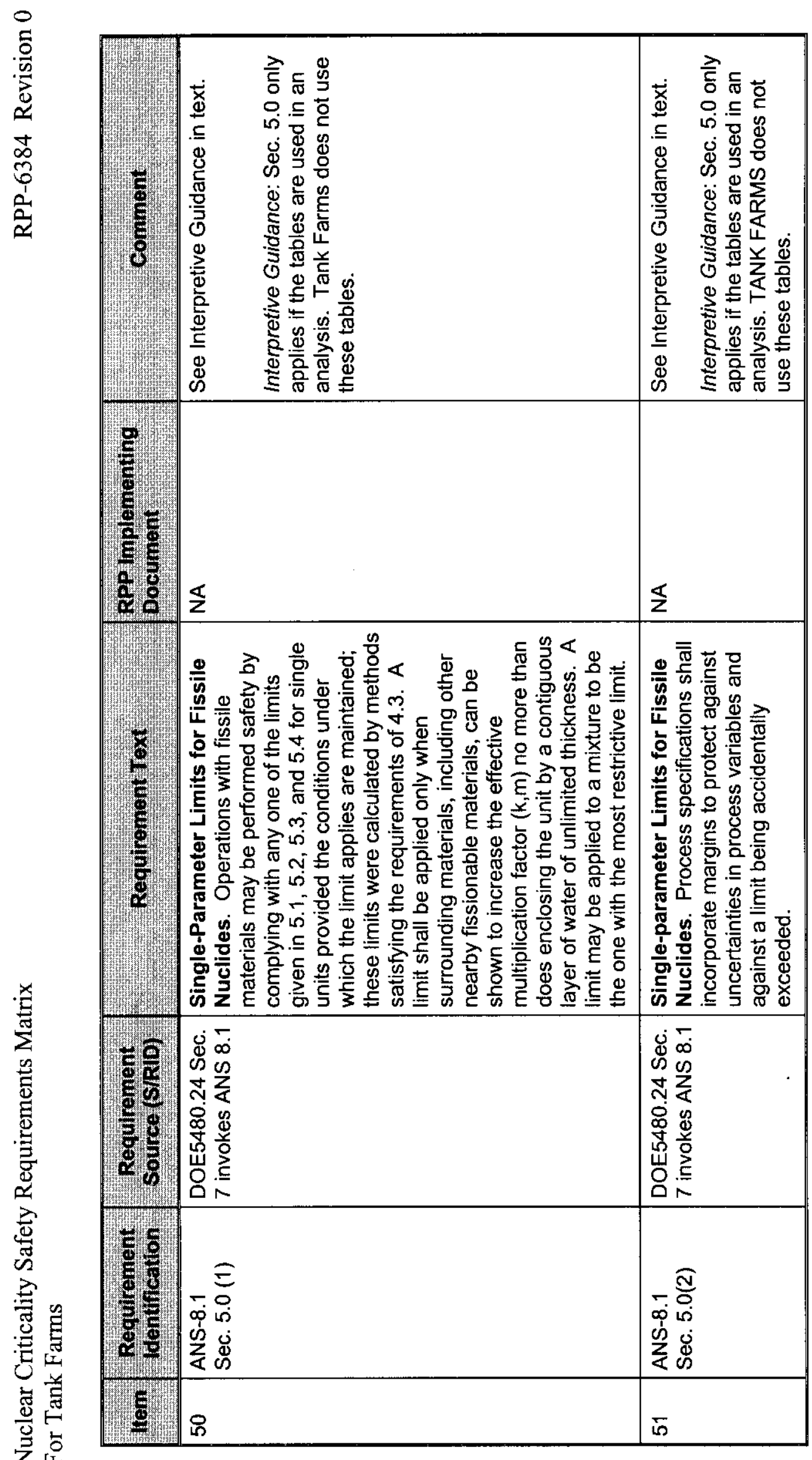




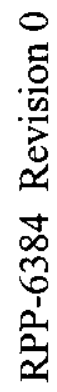

\begin{tabular}{|c|c|c|c|c|}
\hline Whan & 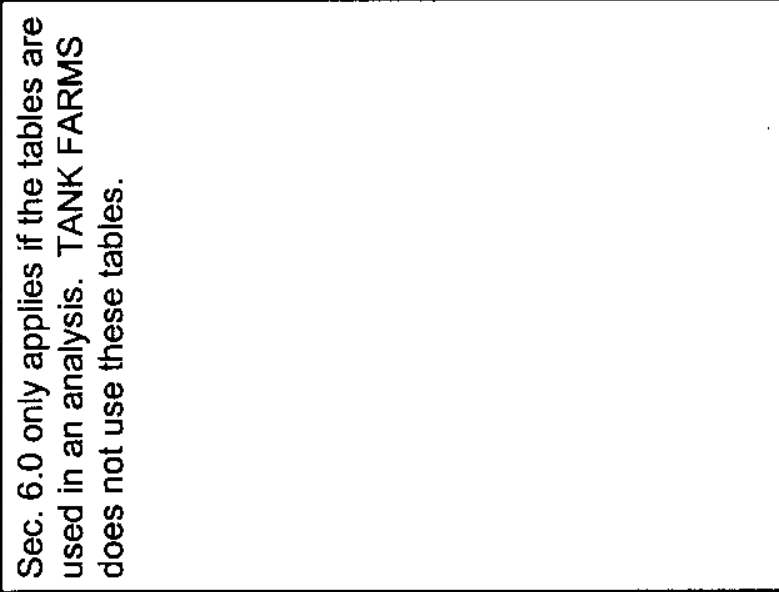 & 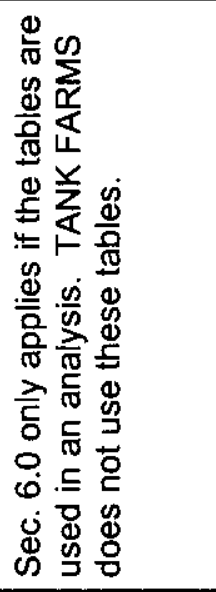 & 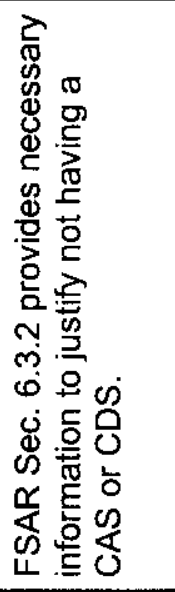 & 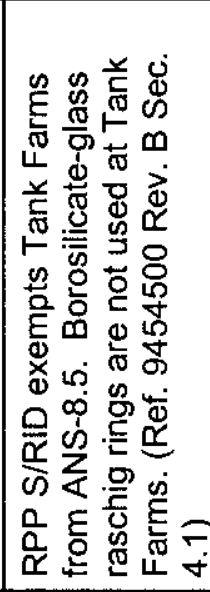 \\
\hline 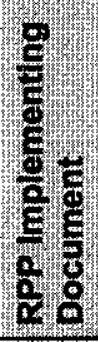 & $\Sigma$ & $\S$ & 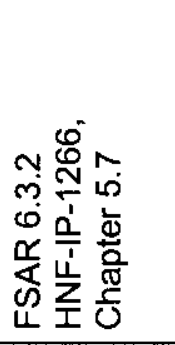 & $\mathbb{Z}$ \\
\hline 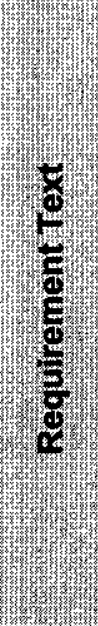 & 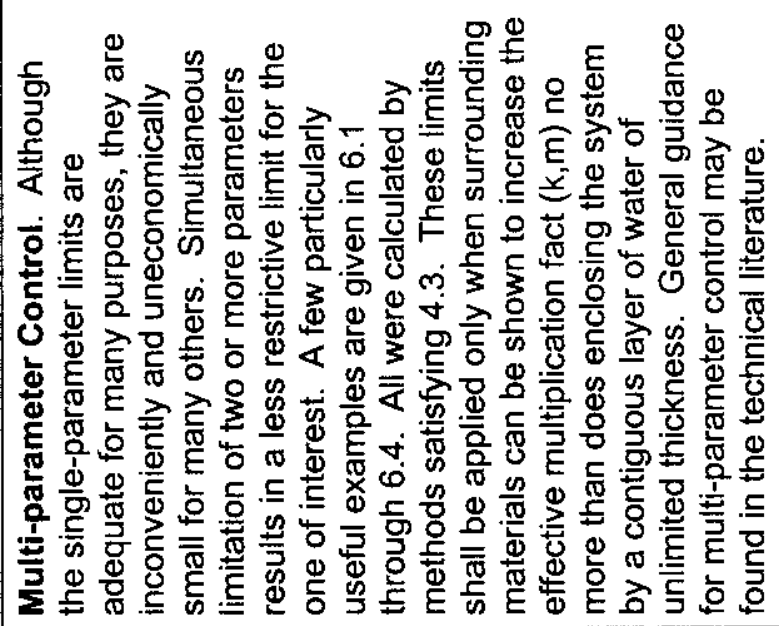 & 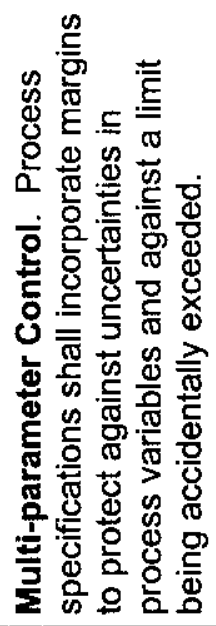 & 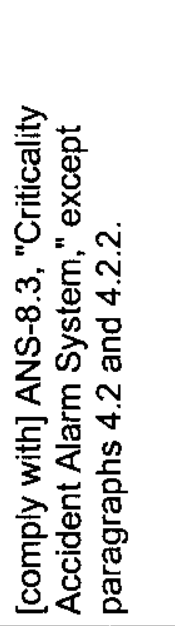 & 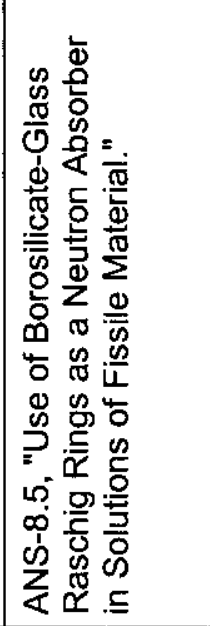 \\
\hline$\frac{6}{6} \frac{a}{6}$ & 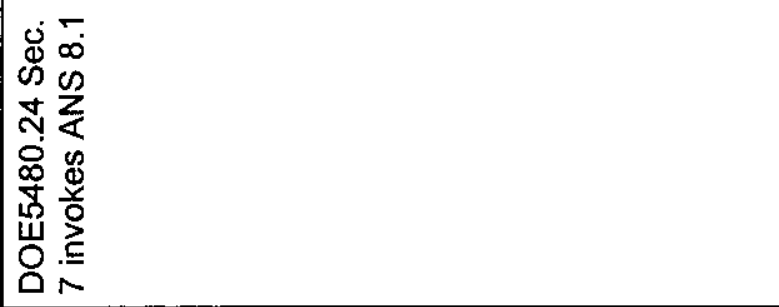 & 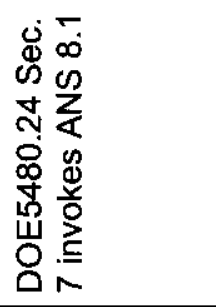 & 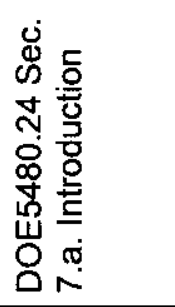 & 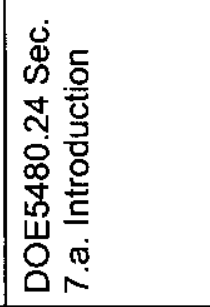 \\
\hline$\frac{5}{9}$ & 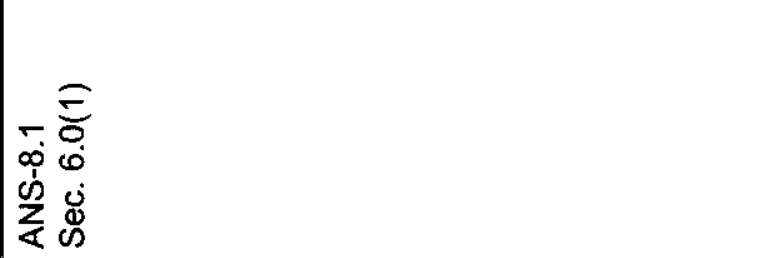 & 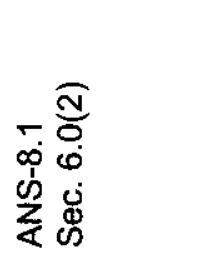 & 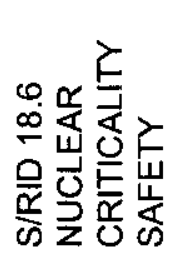 & 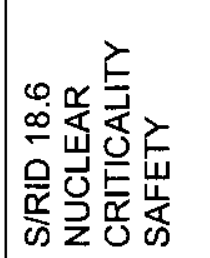 \\
\hline I & గ్ & గి & 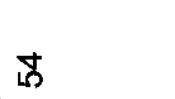 & in \\
\hline
\end{tabular}




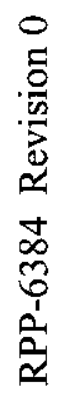

\begin{tabular}{|c|c|c|c|c|c|}
\hline Fe & 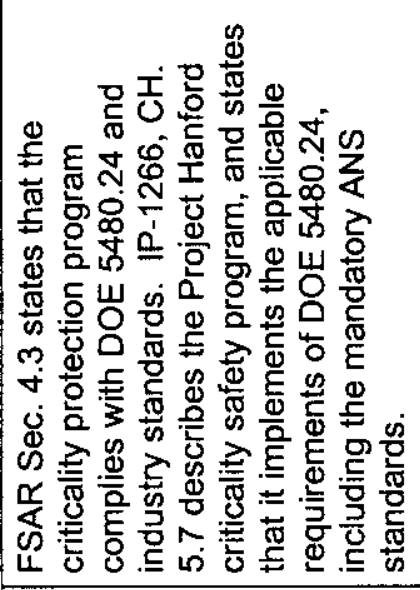 & & & & 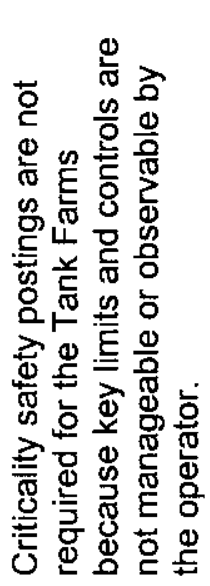 \\
\hline 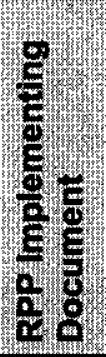 & 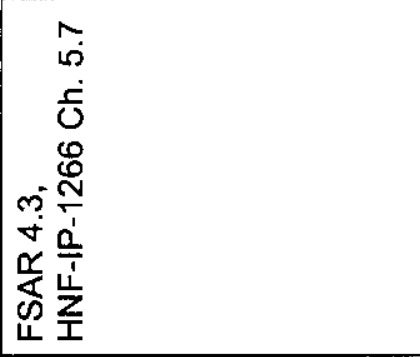 & 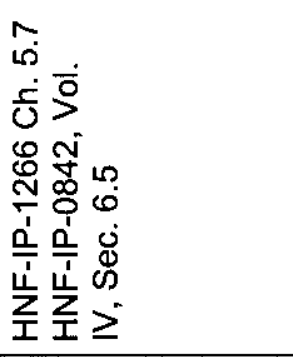 & 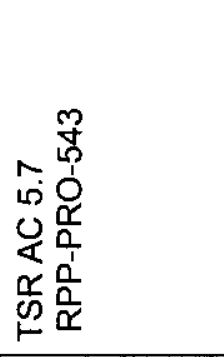 & 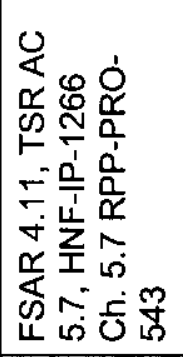 & 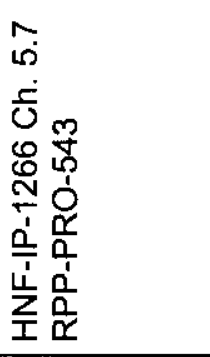 \\
\hline 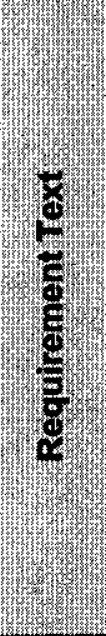 & 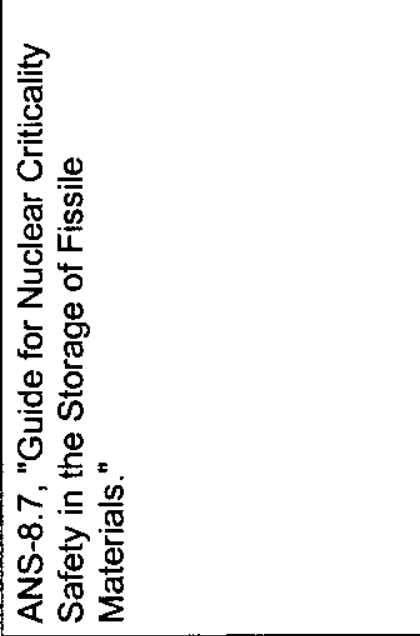 & 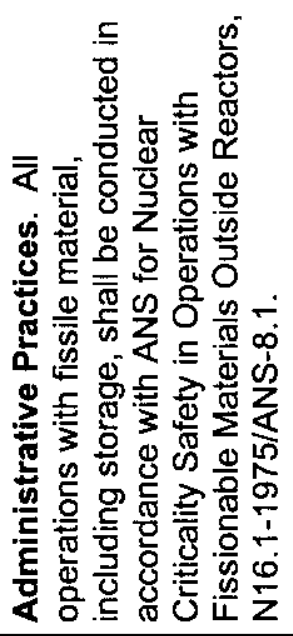 & 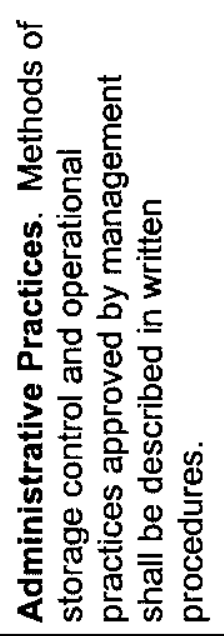 & 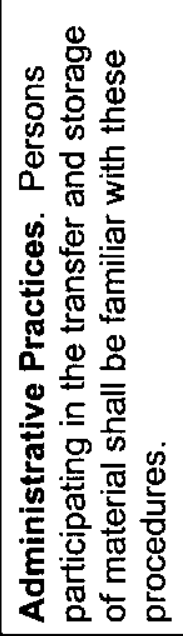 & 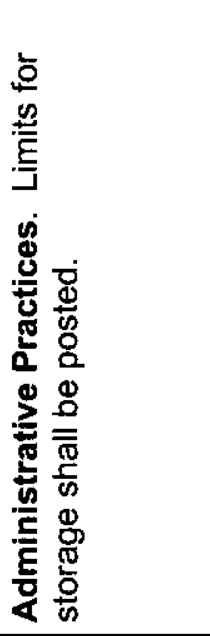 \\
\hline$\frac{g}{6} \frac{9}{6}$ & 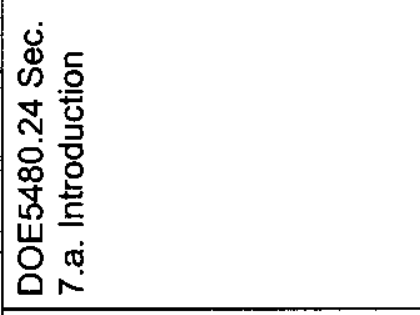 & 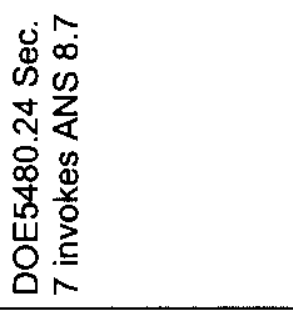 & 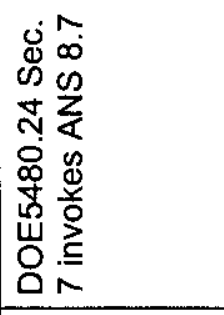 & 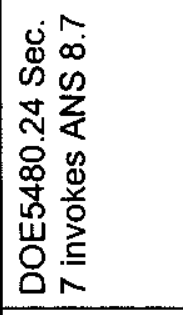 & 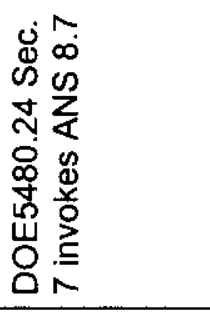 \\
\hline 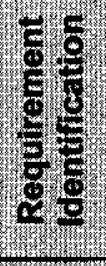 & 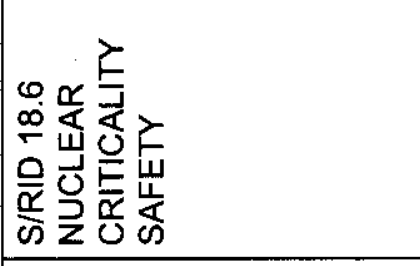 & 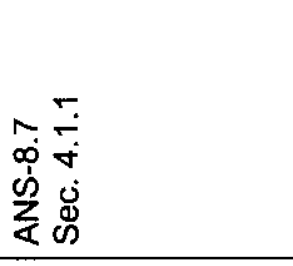 & 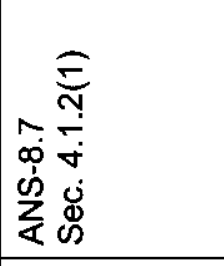 & 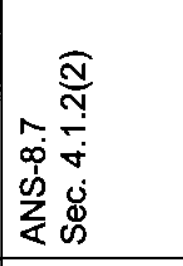 & 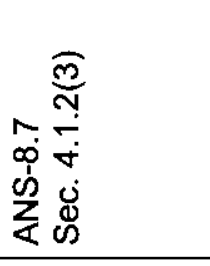 \\
\hline E & : & to & 曲 & (9) & 8 \\
\hline
\end{tabular}




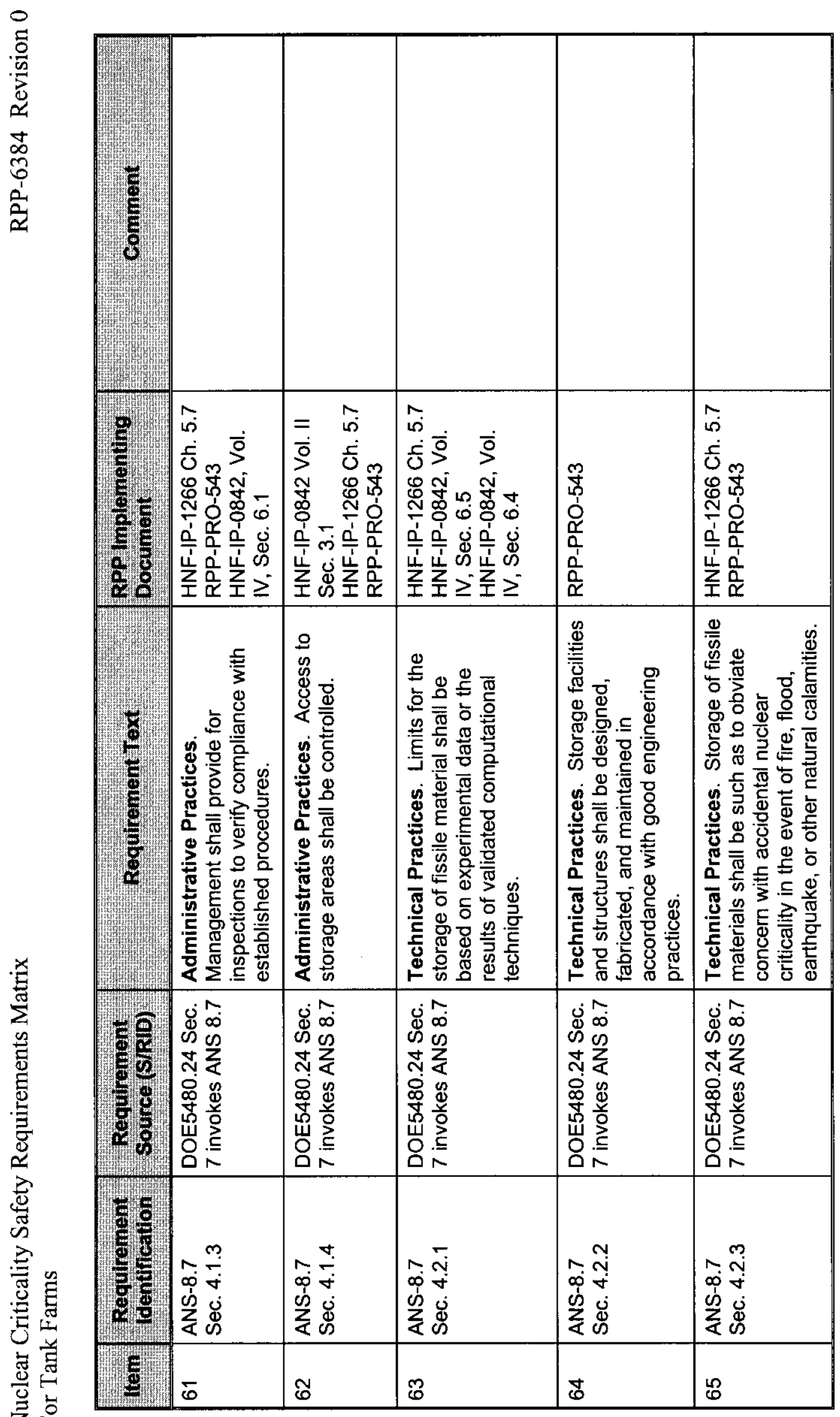




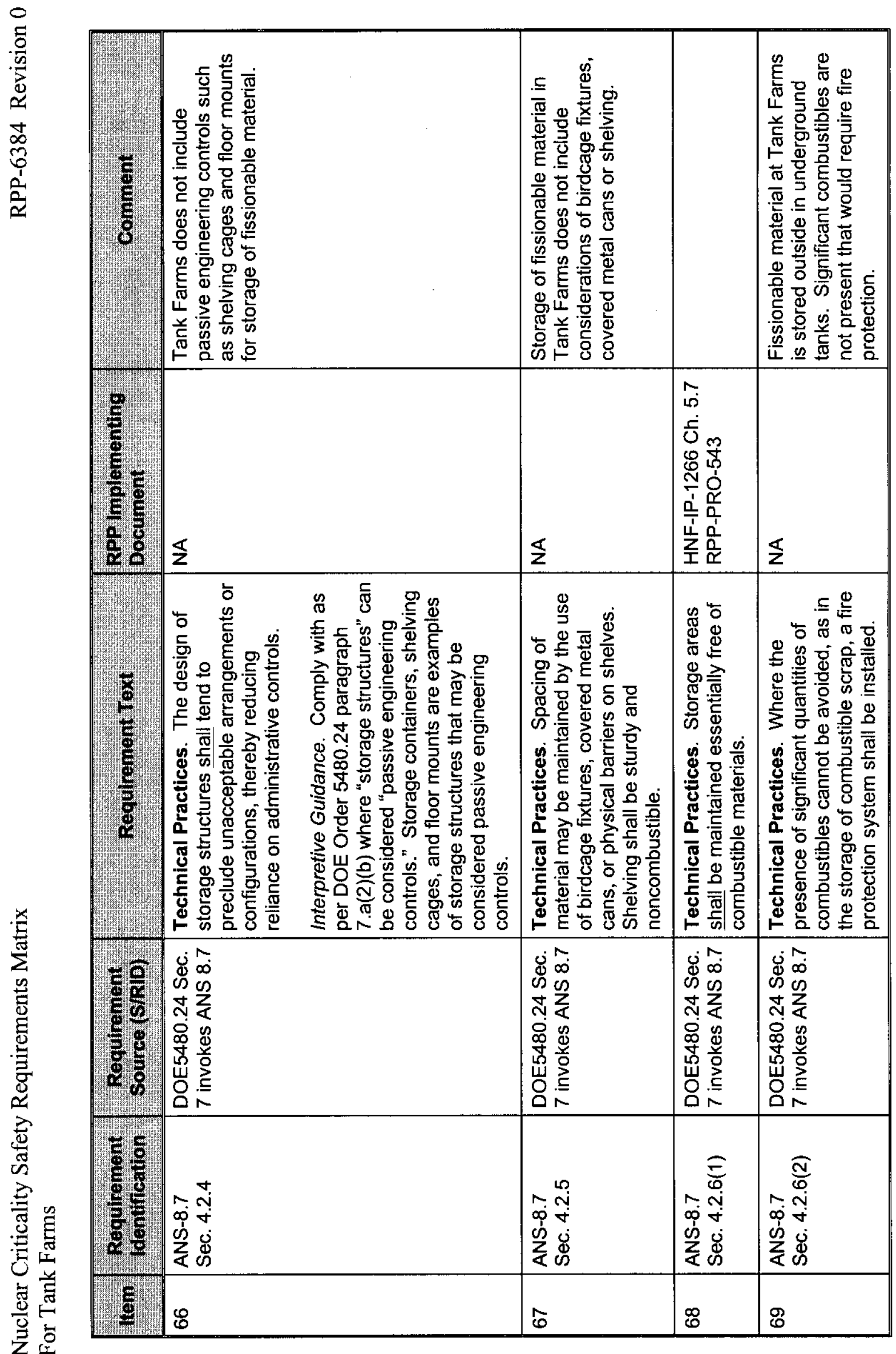




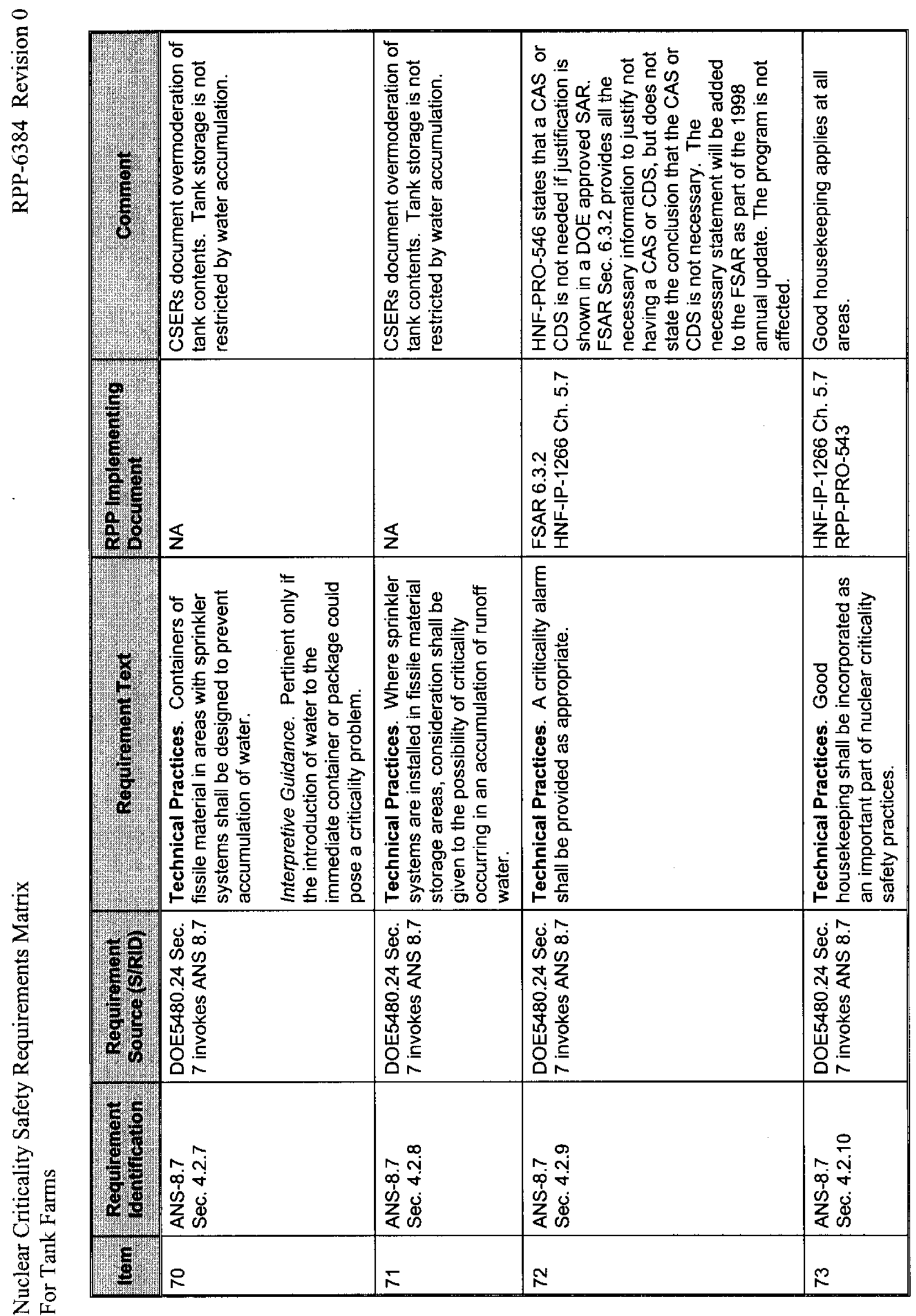




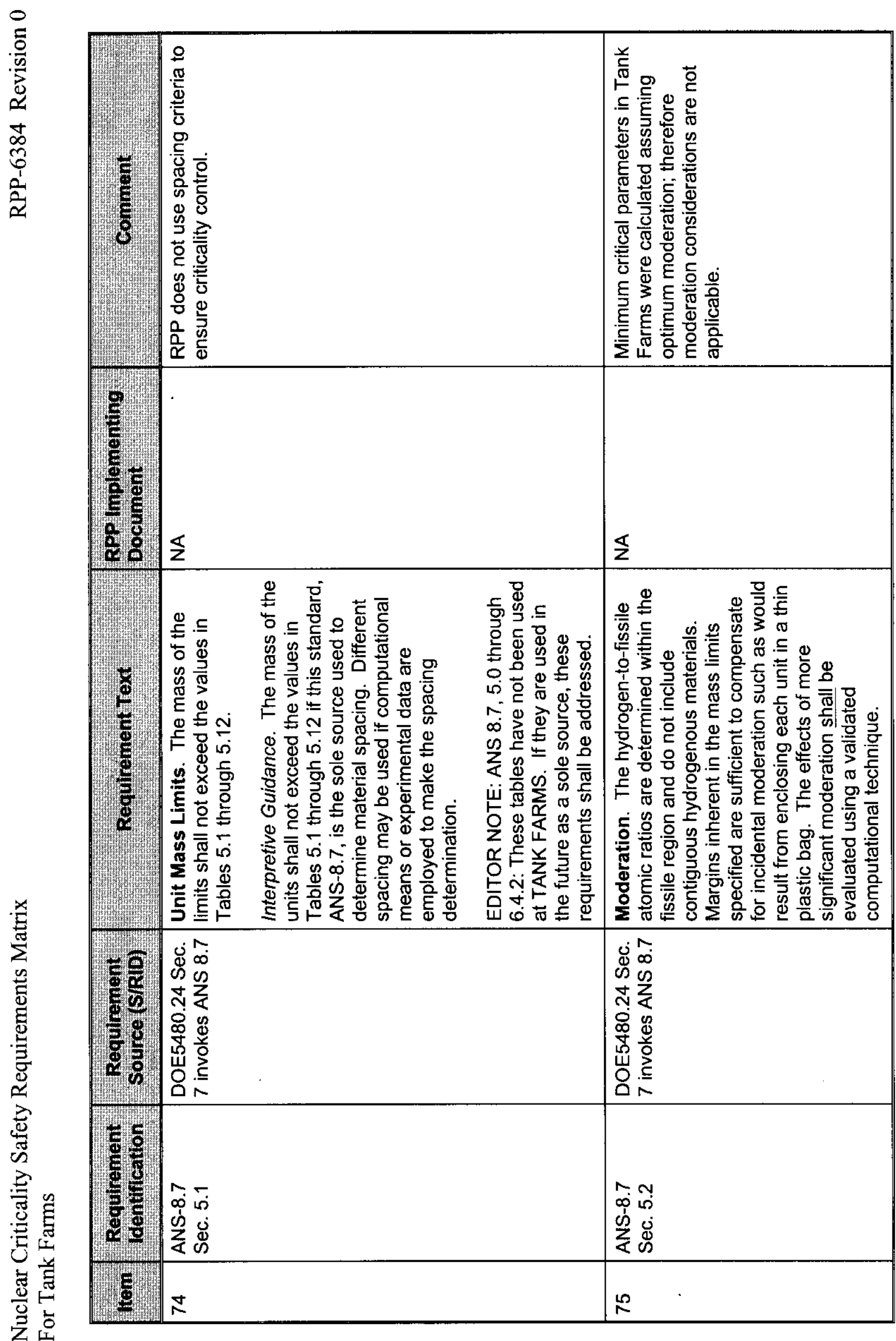




\begin{tabular}{|c|c|c|c|c|}
\hline 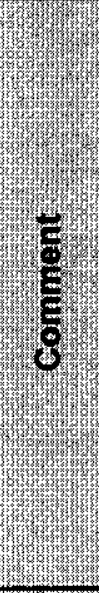 & 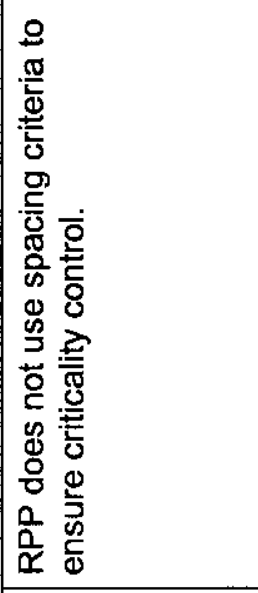 & 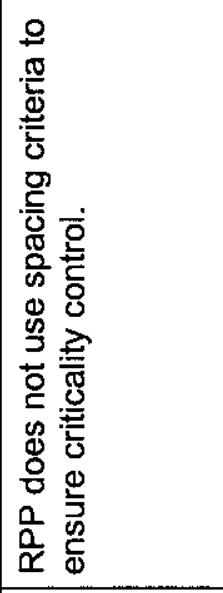 & 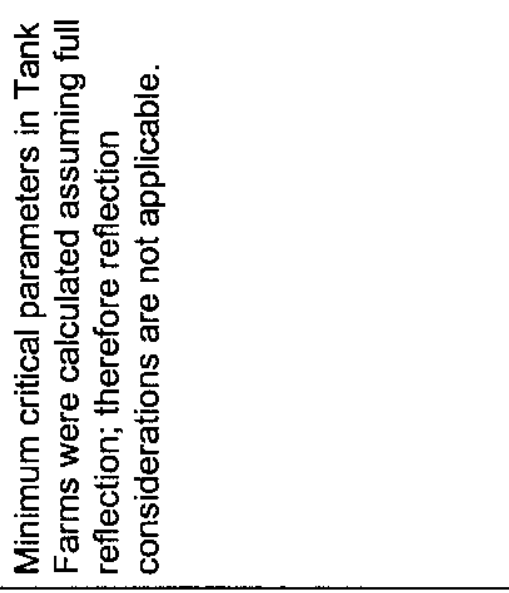 & 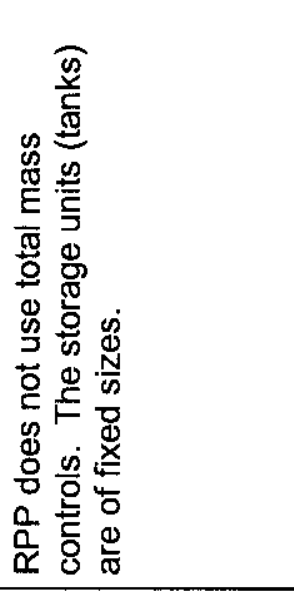 \\
\hline 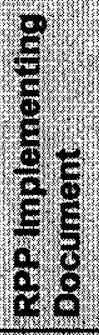 & 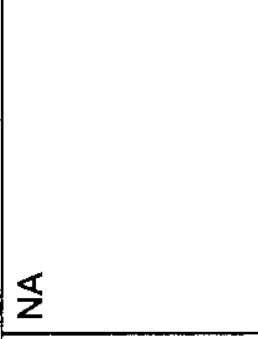 & $\Sigma$ & $\Sigma$ & $\overleftarrow{z}$ \\
\hline${ }^{2}$ & 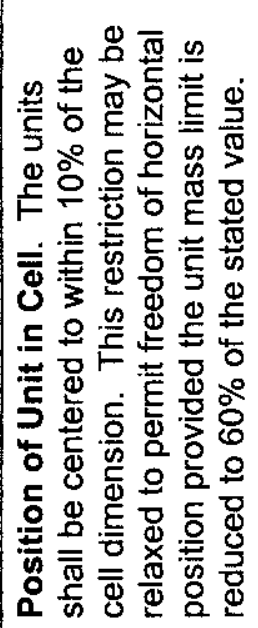 & 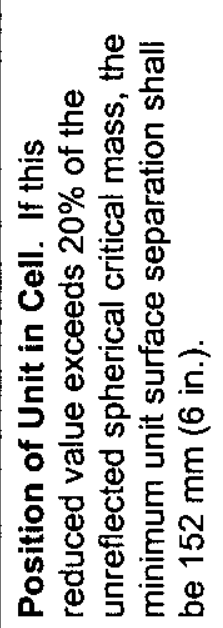 & 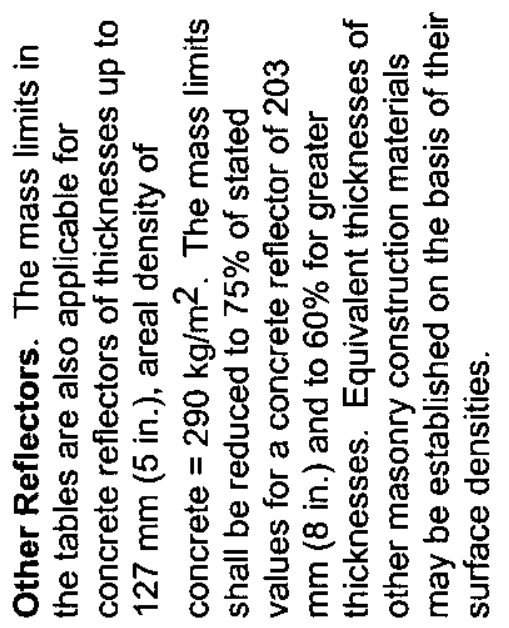 & 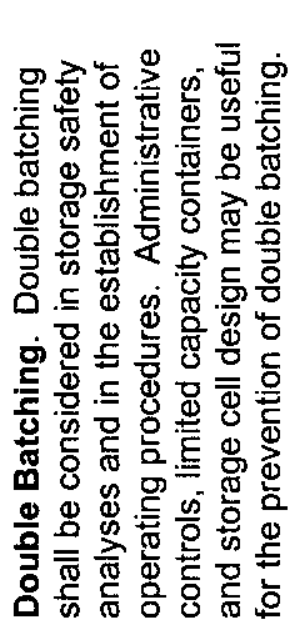 \\
\hline 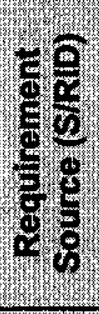 & 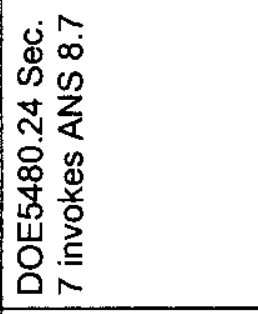 & 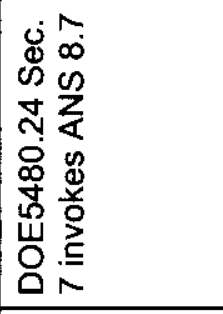 & 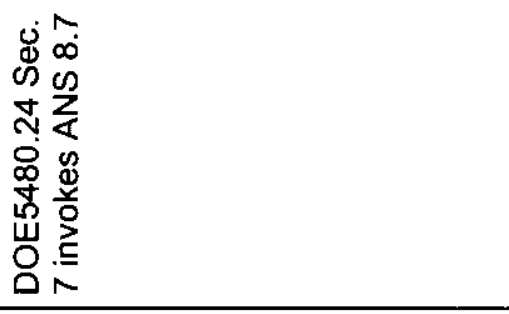 & 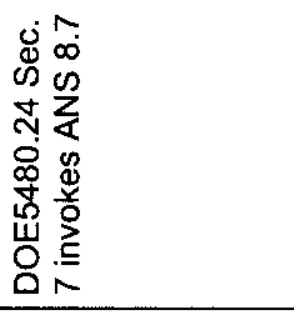 \\
\hline $\begin{array}{l}5 \\
5 \\
8 \\
8\end{array}$ & 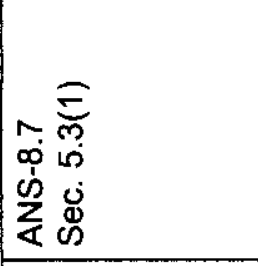 & 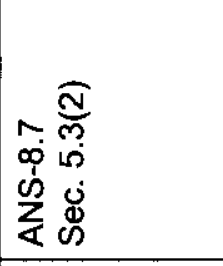 & 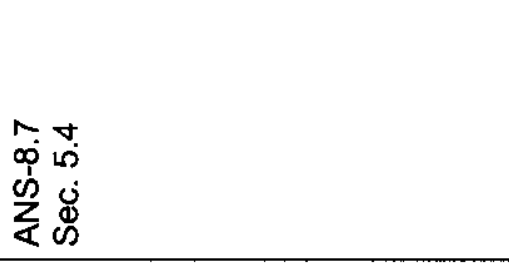 & 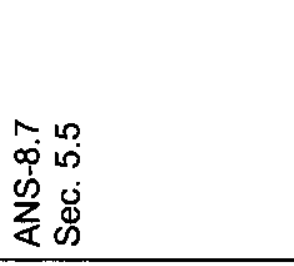 \\
\hline 9 & 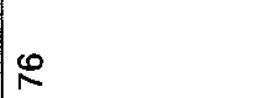 & 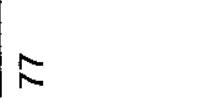 & $\stackrel{\infty}{\sim}$ & $R$ \\
\hline
\end{tabular}




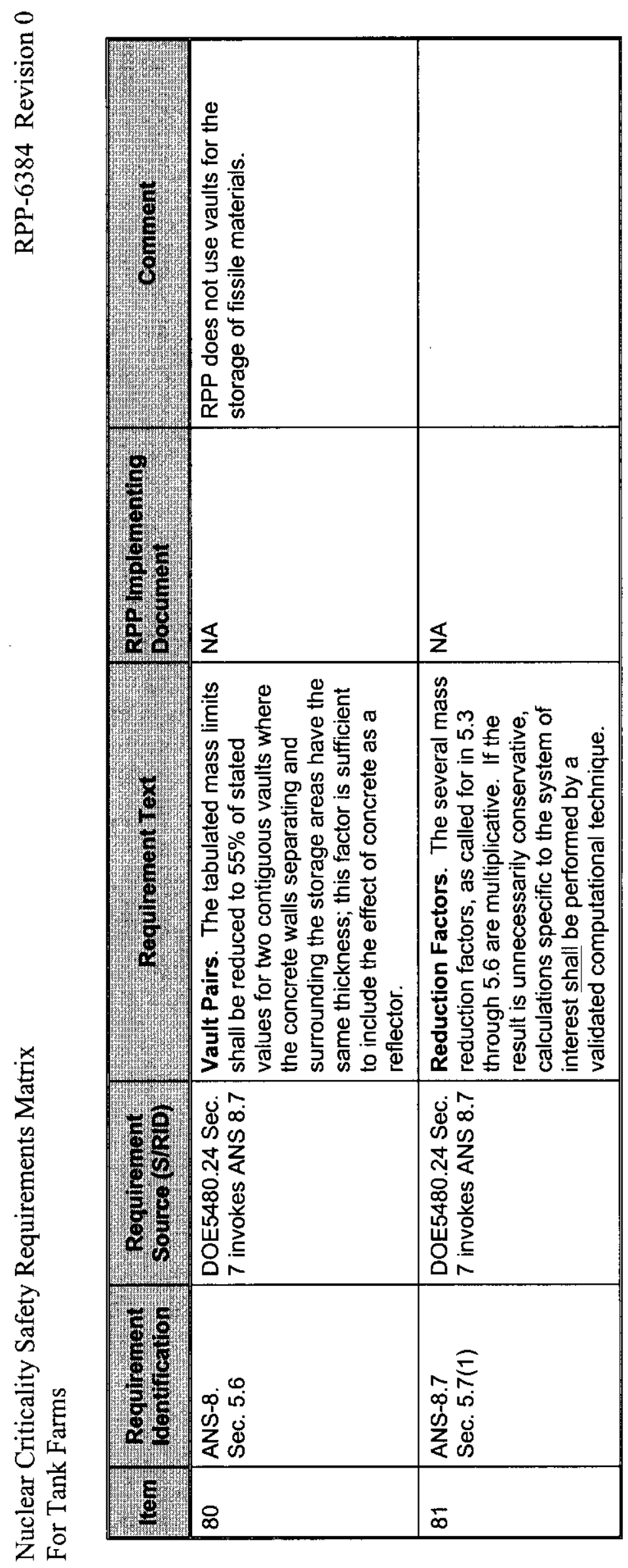




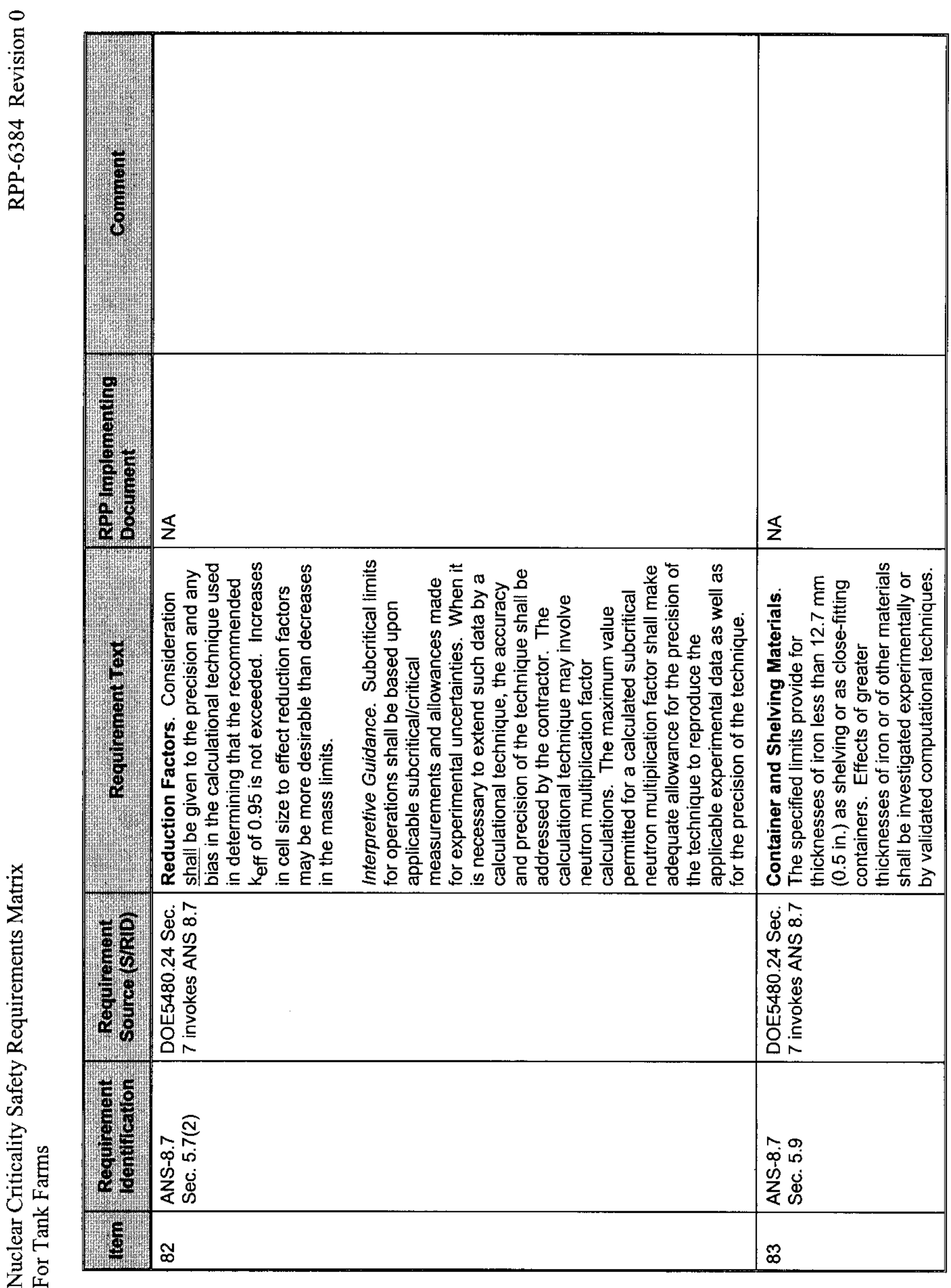

จ 


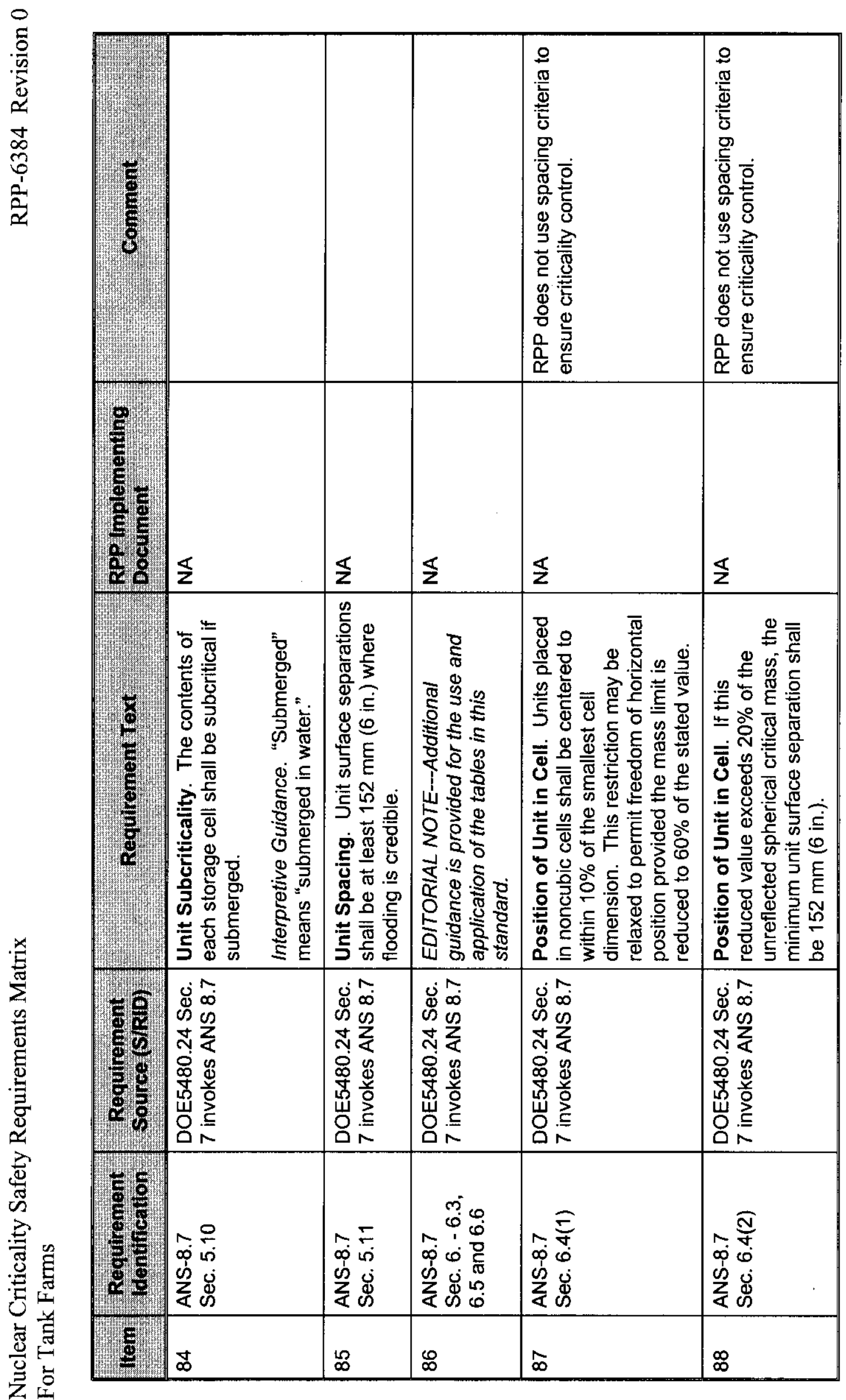




\begin{tabular}{|c|c|c|c|c|c|}
\hline 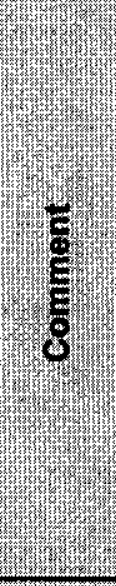 & 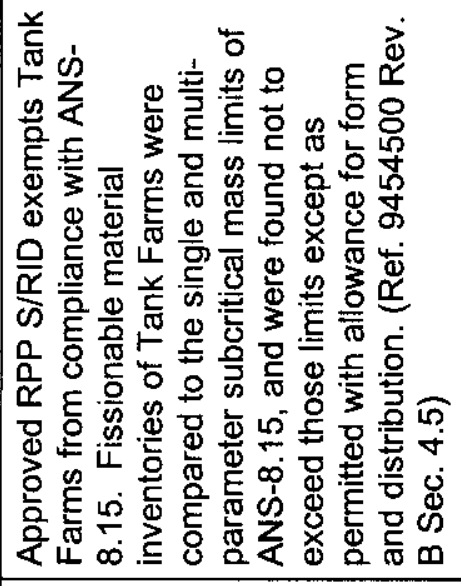 & 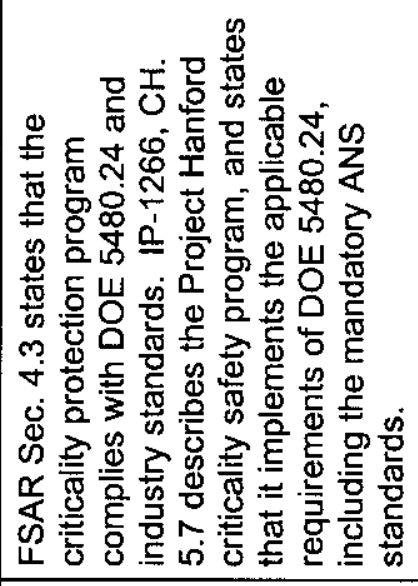 & 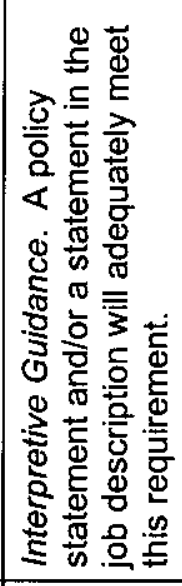 & & \\
\hline 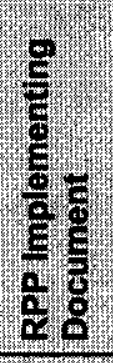 & 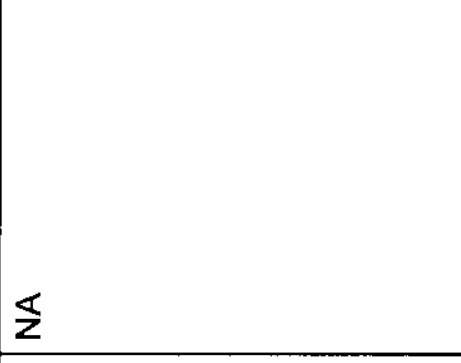 & 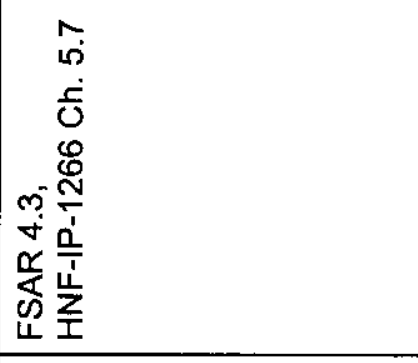 & 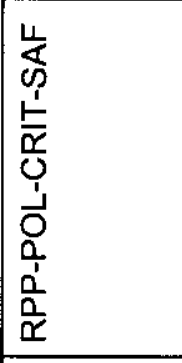 & 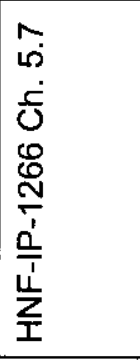 & 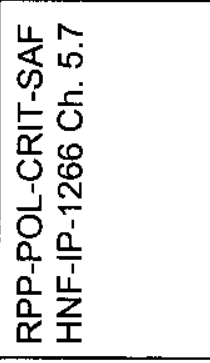 \\
\hline$\frac{F}{2}$ & 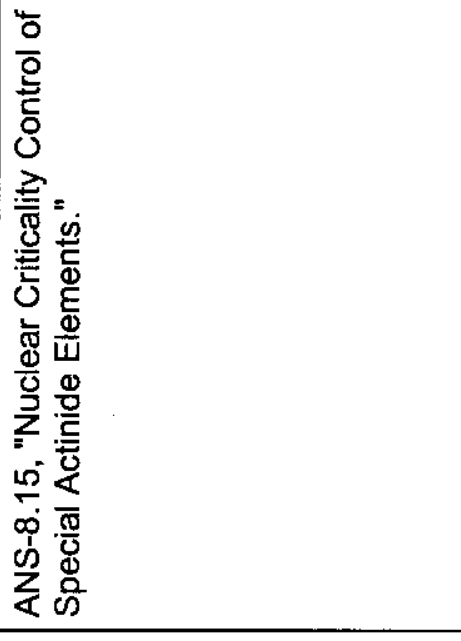 & 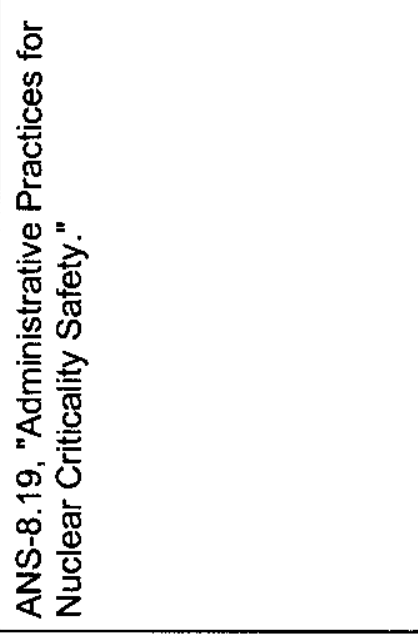 & 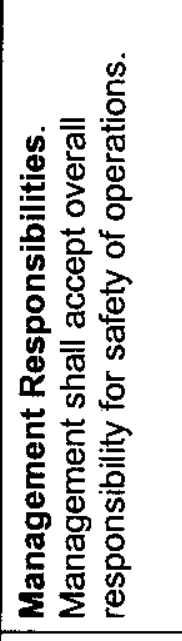 & 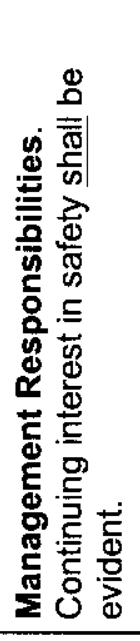 & 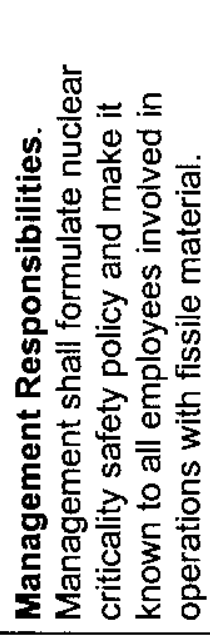 \\
\hline$\frac{5}{8} \frac{6}{6}$ & 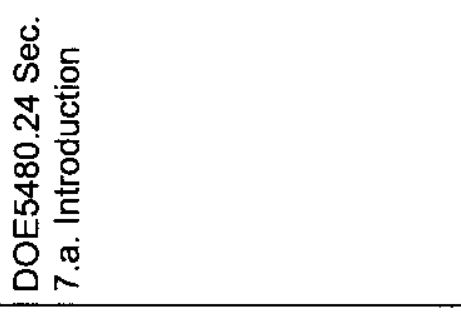 & 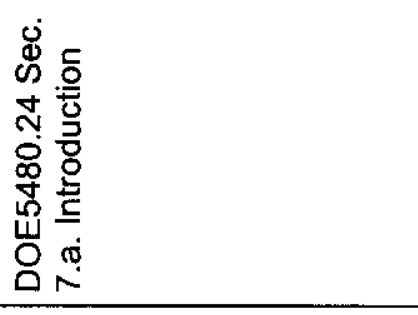 & 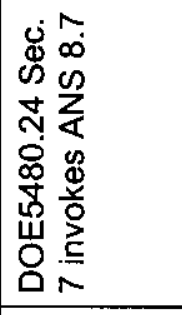 & 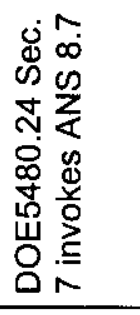 & 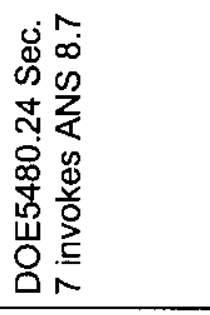 \\
\hline$\frac{9}{8}$ & 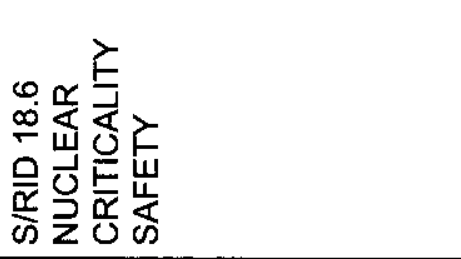 & 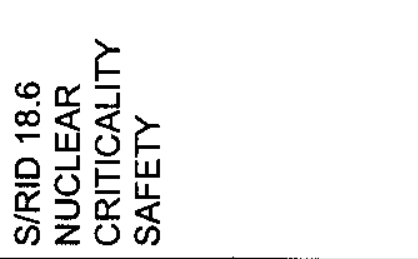 & 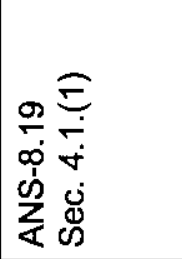 & 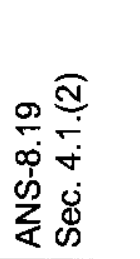 & 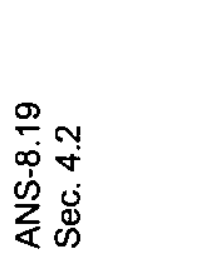 \\
\hline$\frac{5}{s}$ & ది & \& & $\bar{\sigma}$ & ชั & $\mathscr{O}$ \\
\hline
\end{tabular}




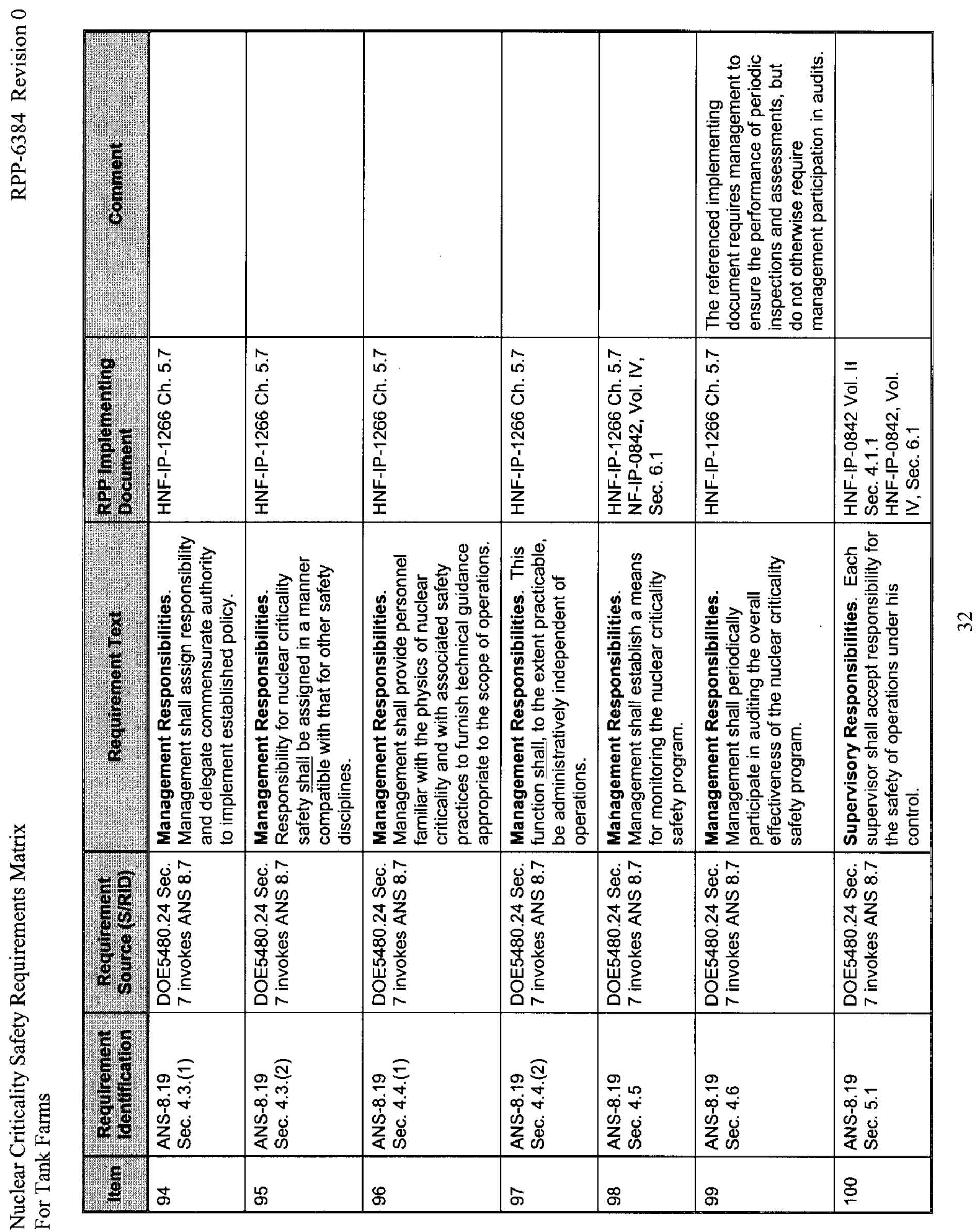




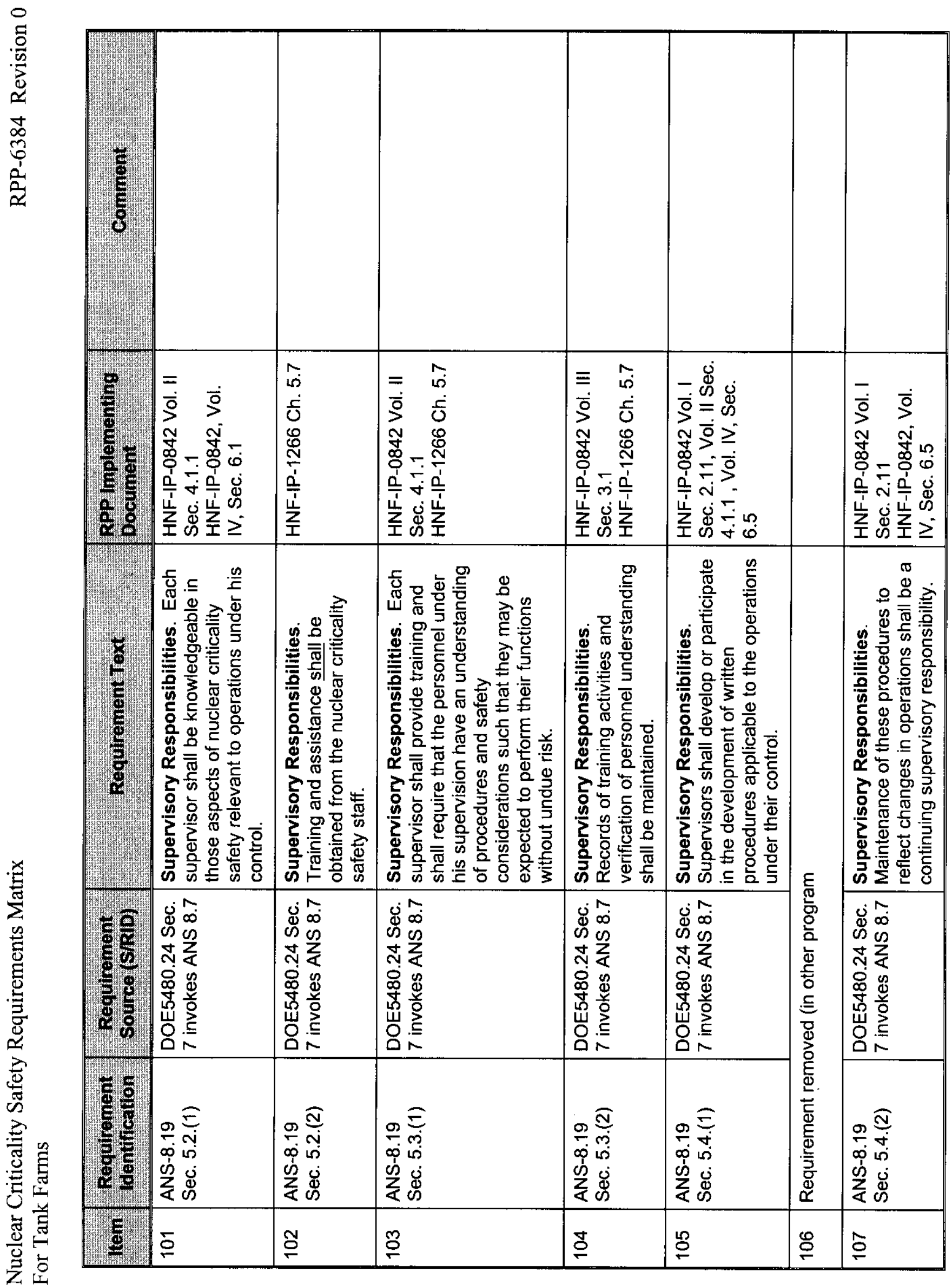


0
0
0
0
0
0
0
5
0
0
0
0
0
01

\begin{tabular}{|c|c|c|c|c|c|c|}
\hline (3) & & & & & \multicolumn{2}{|l|}{ 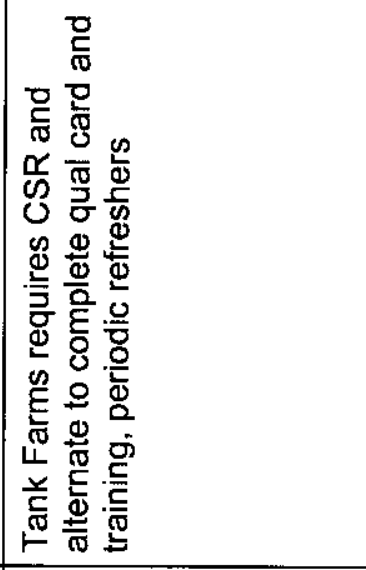 } \\
\hline 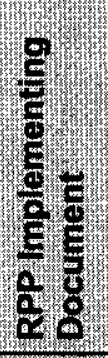 & 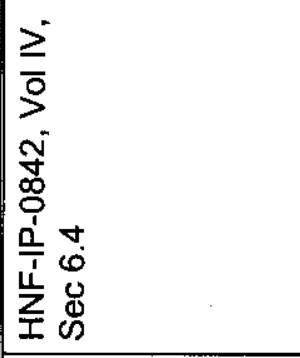 & 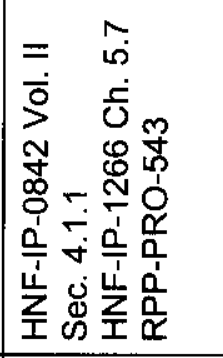 & 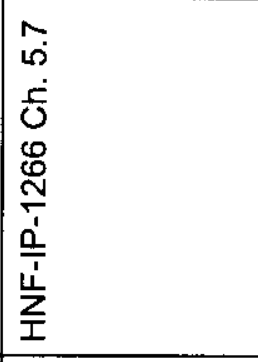 & 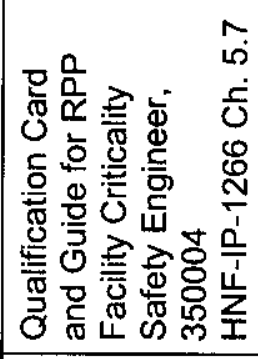 & 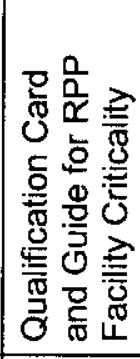 & 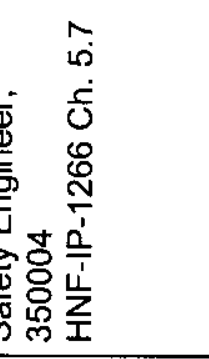 \\
\hline$\frac{8}{\frac{8}{6}}$ & 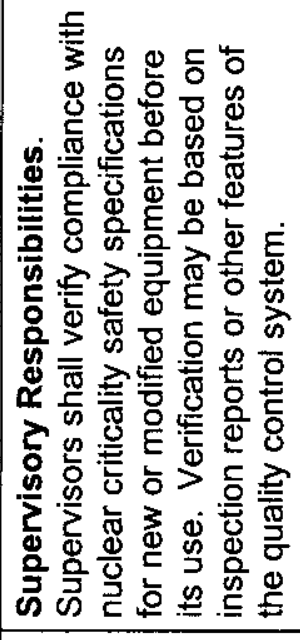 & 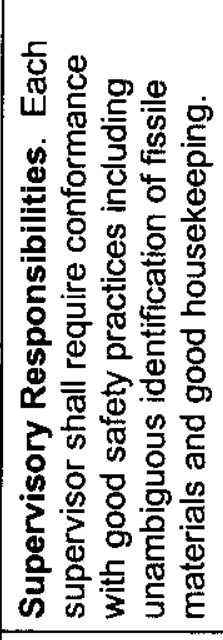 & 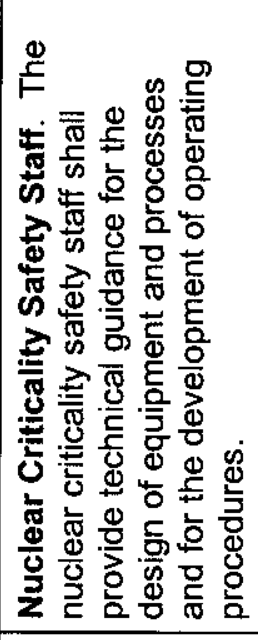 & 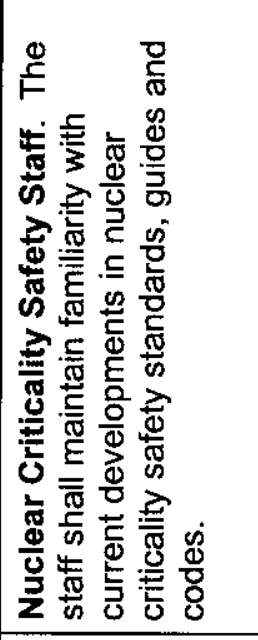 & 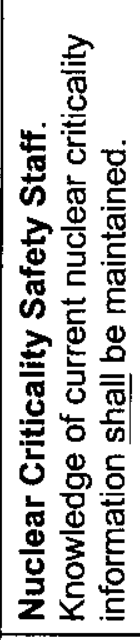 & 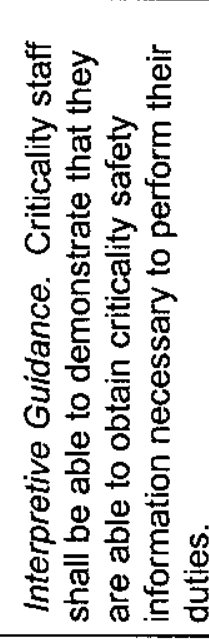 \\
\hline 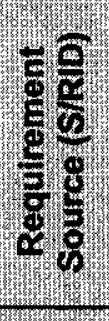 & 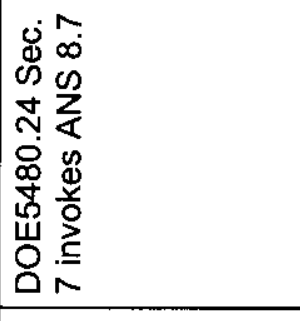 & 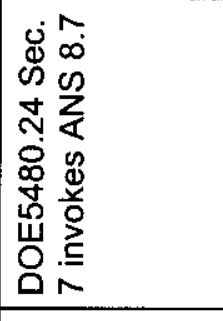 & 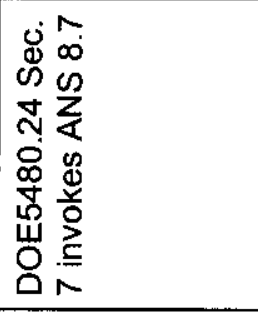 & 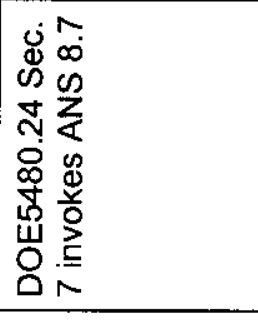 & 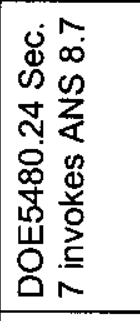 & \\
\hline$\frac{7}{8}$ & 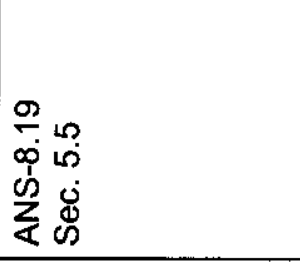 & 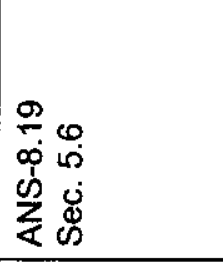 & 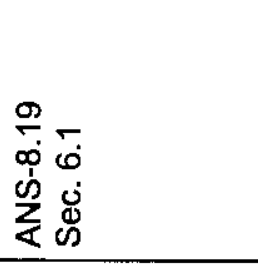 & 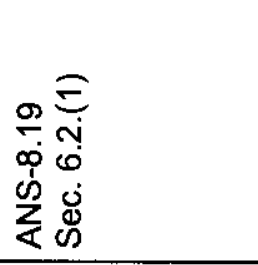 & 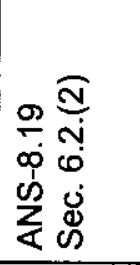 & \\
\hline 8 & $\stackrel{\infty}{\circ}$ & $\stackrel{9}{\circ}$ & $\stackrel{ }{ }$ & $\check{F}$ & $\stackrel{\mathbb{N}}{\leftarrow}$ & \\
\hline
\end{tabular}




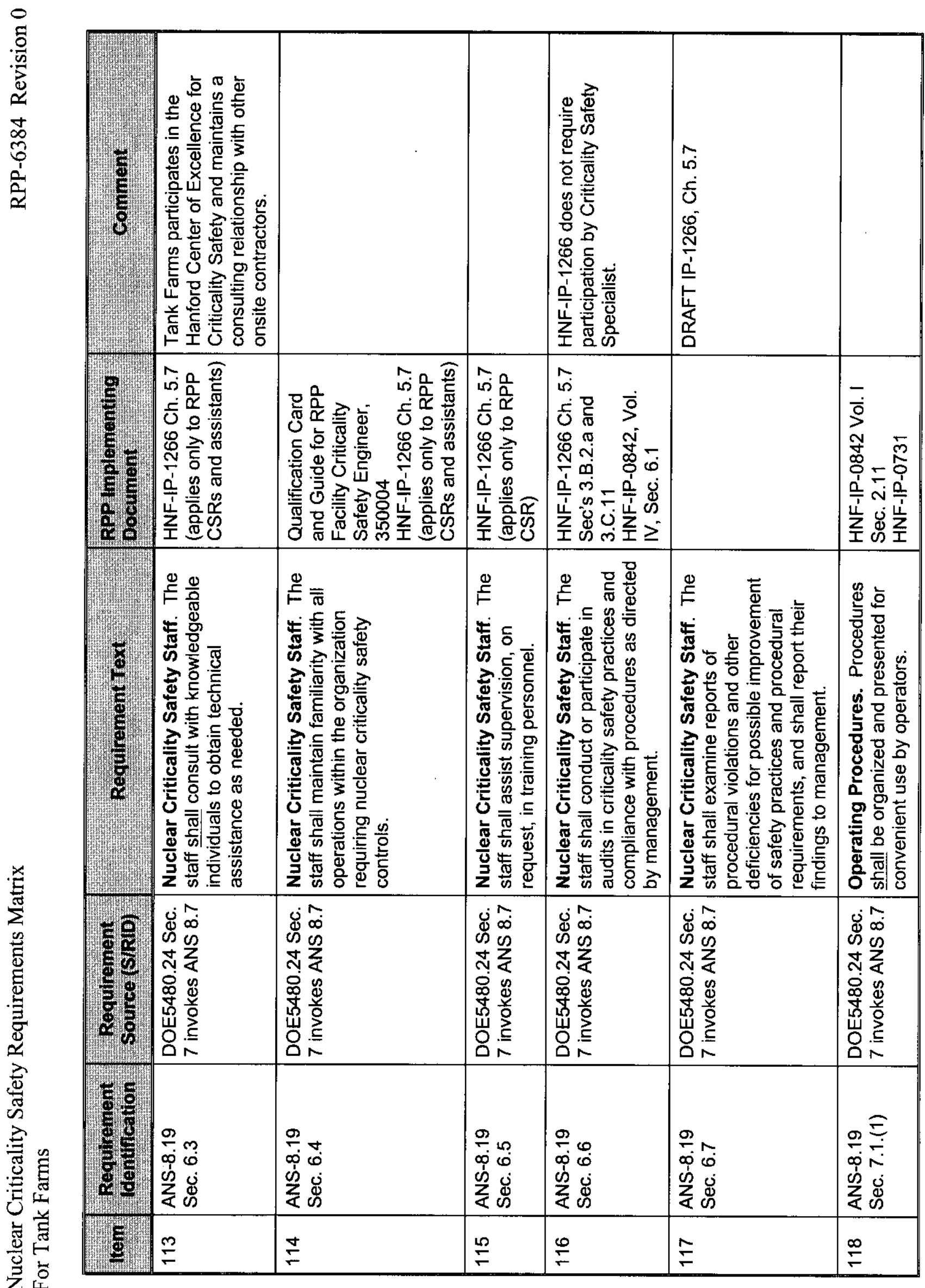


0
1
0
$\frac{0}{2}$
0
$\simeq$
5
$\infty$
00
01
01
2

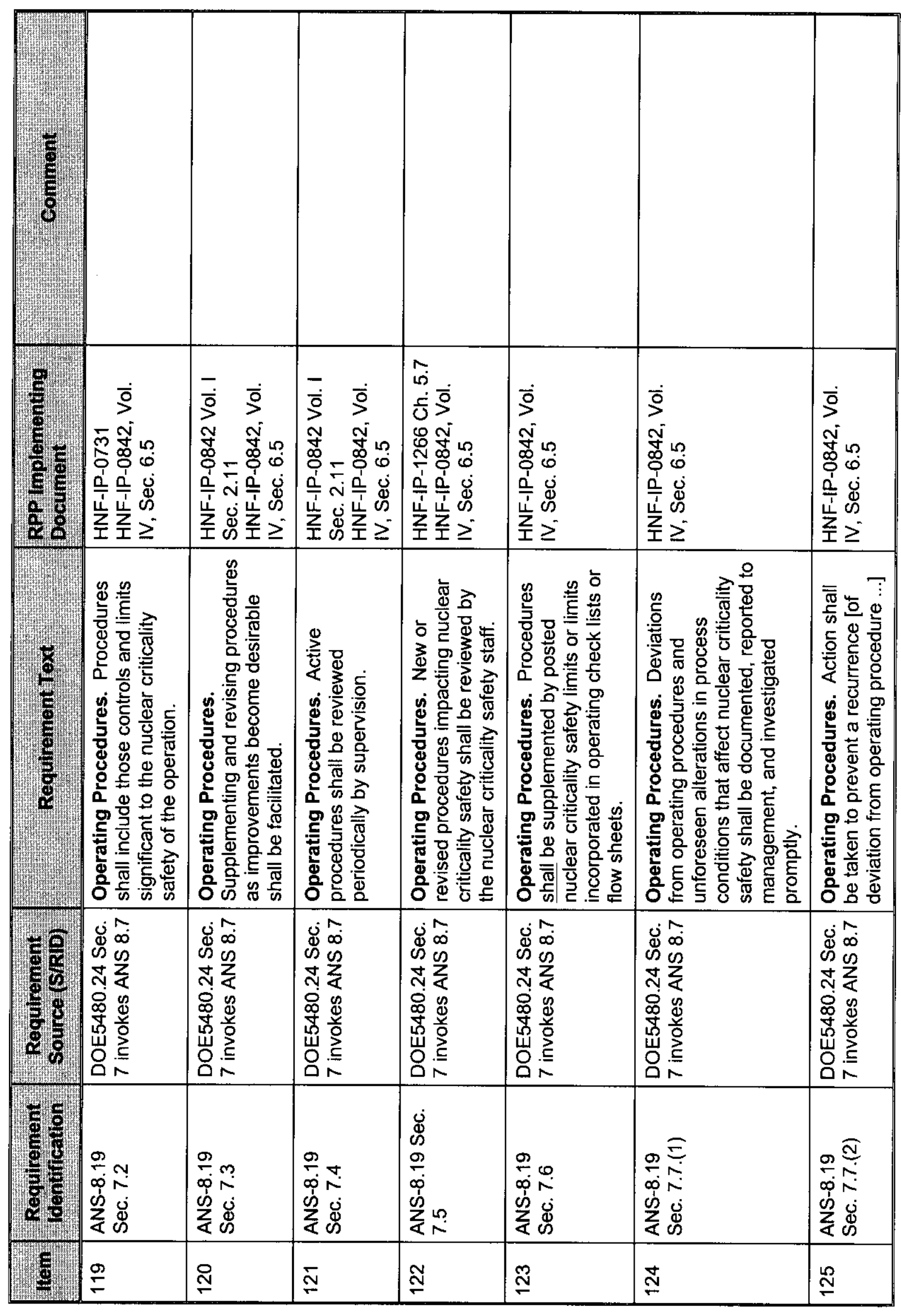




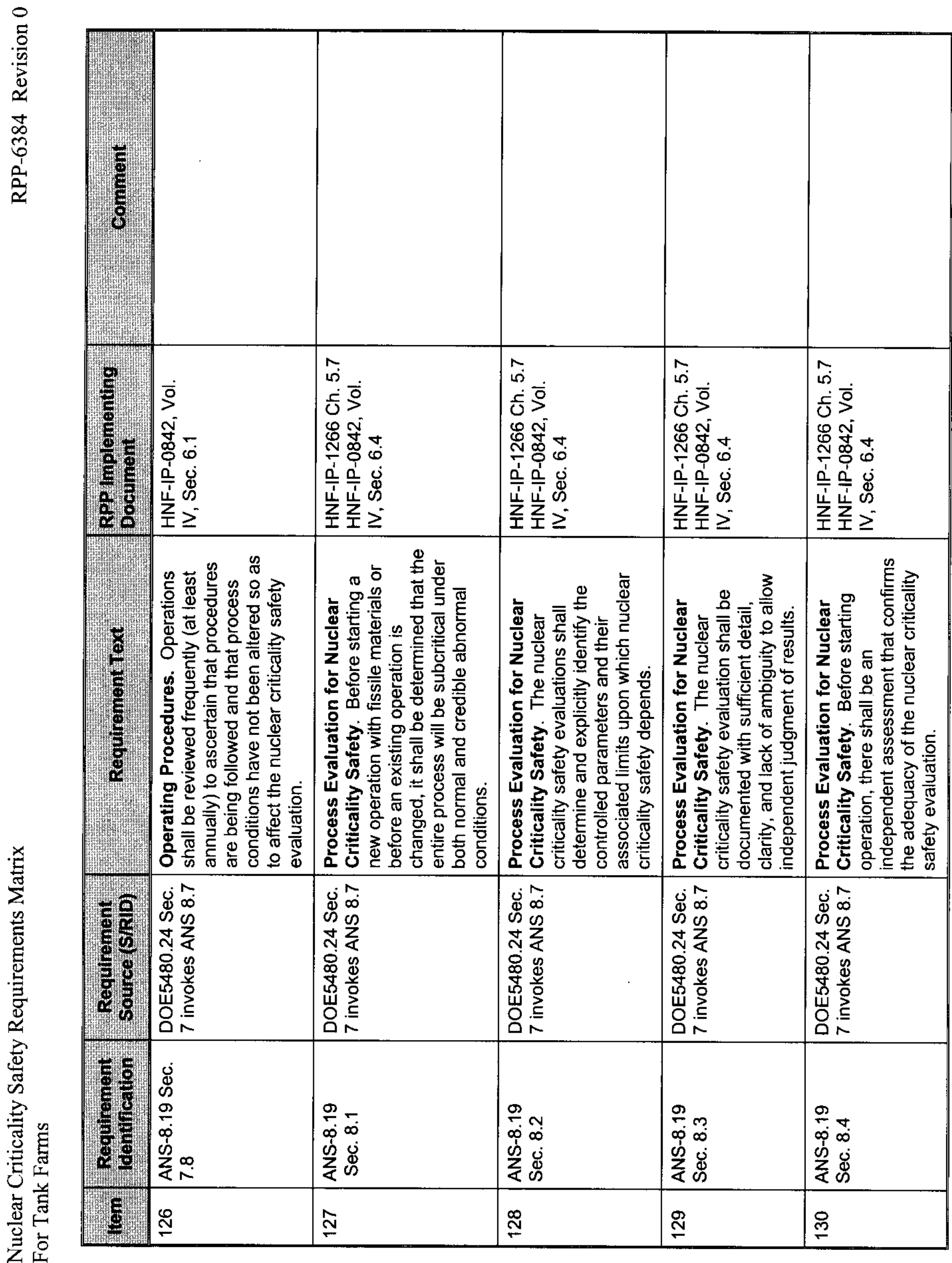

$n$ 


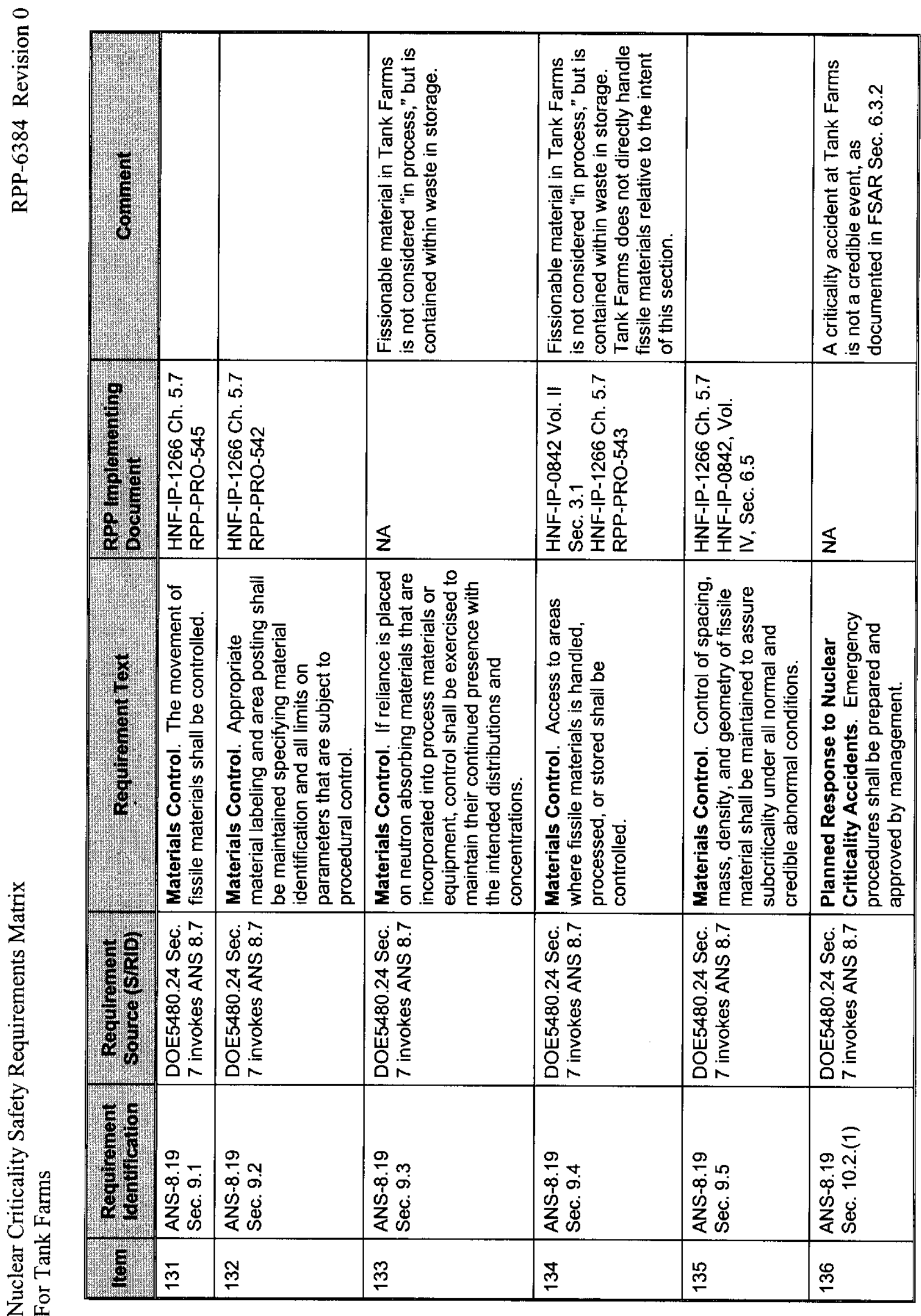




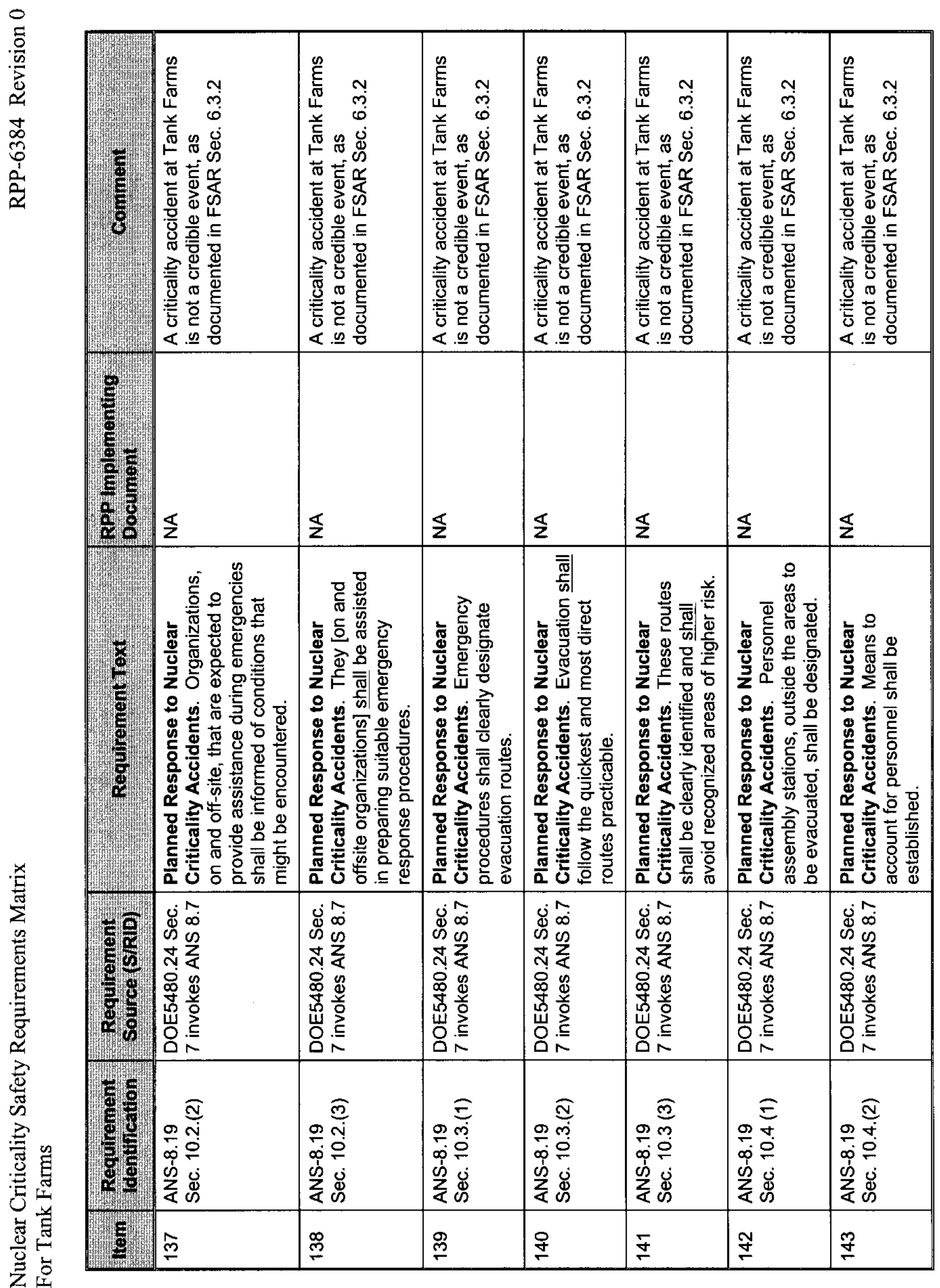

9 


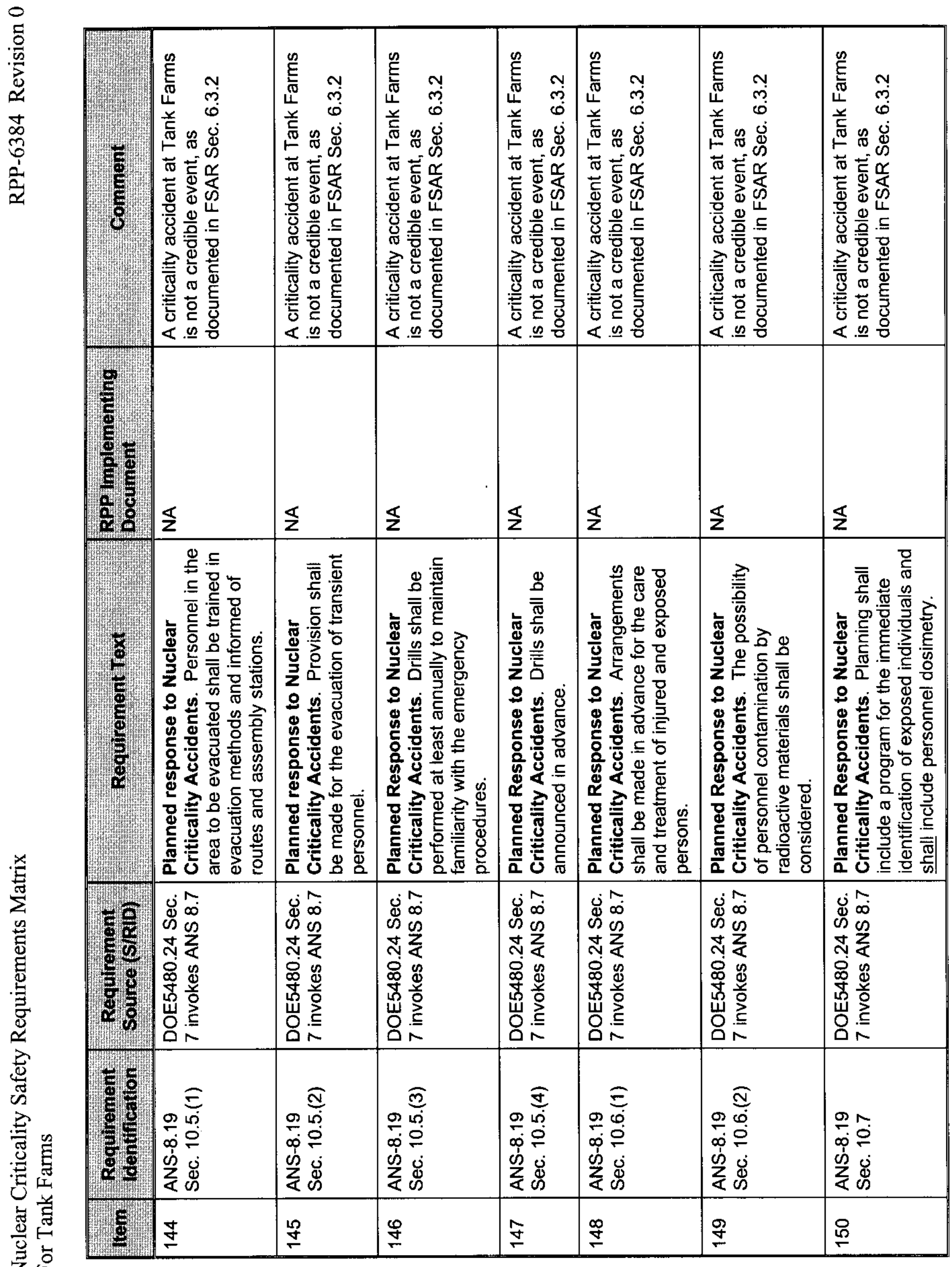




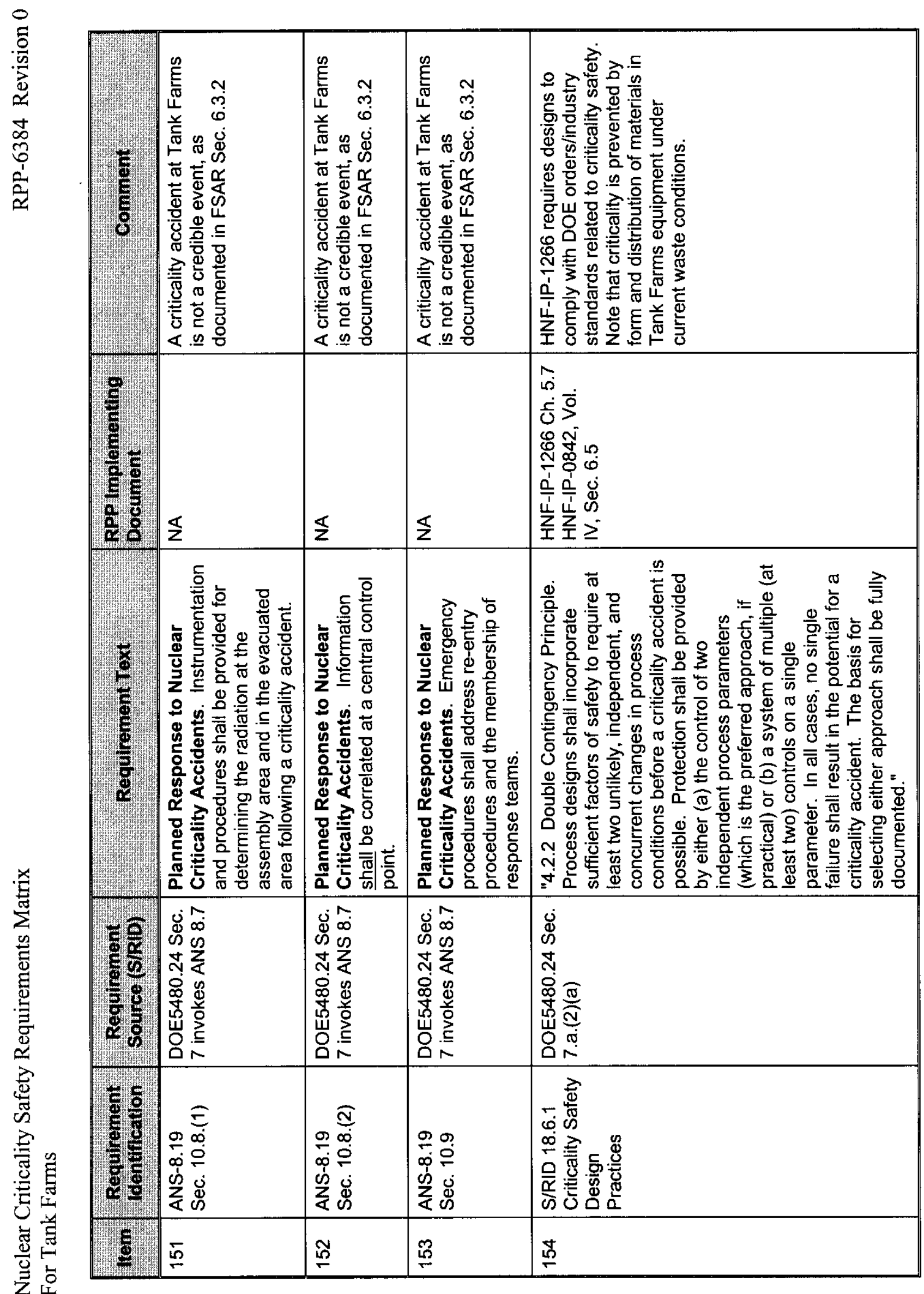




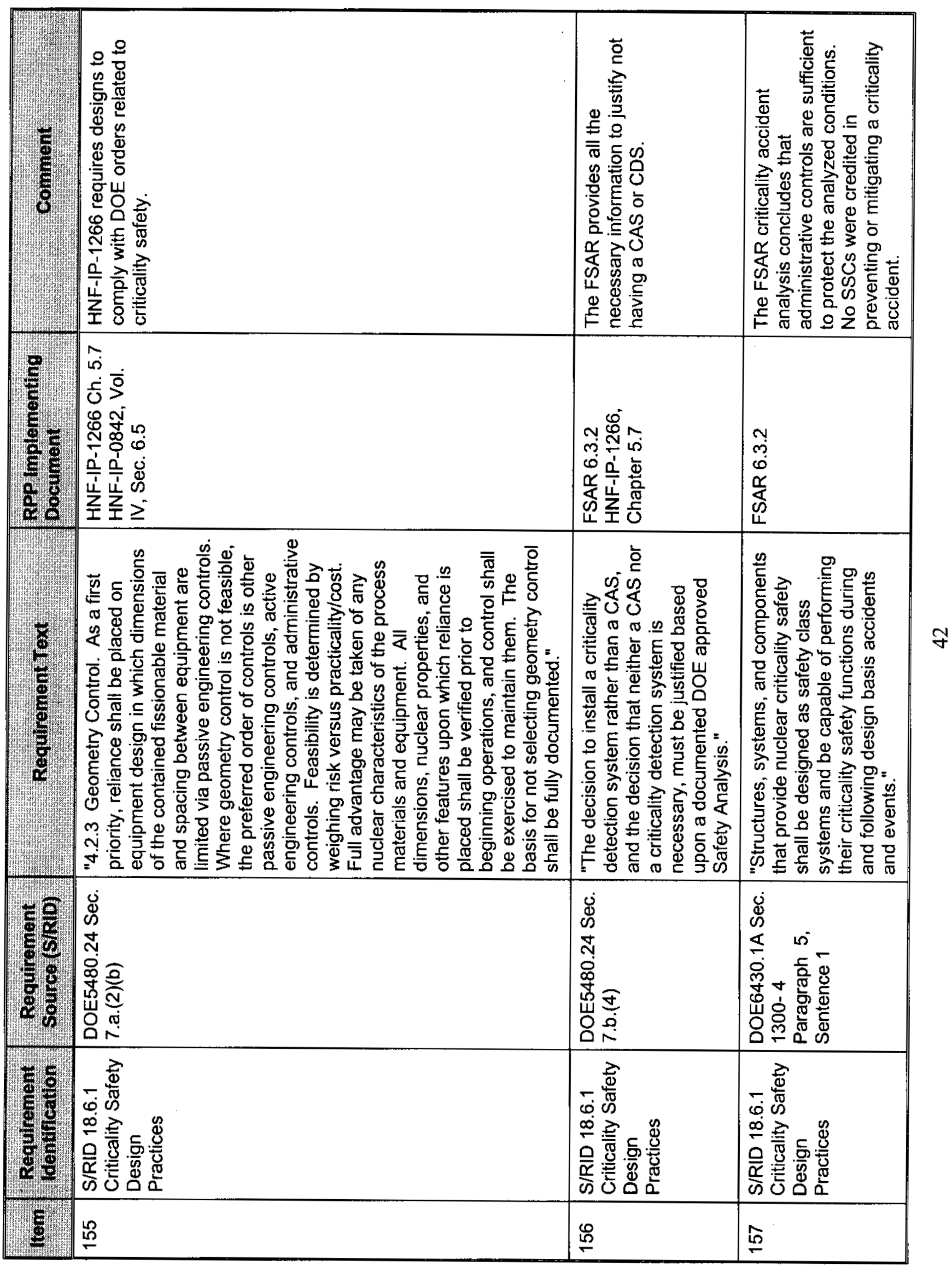




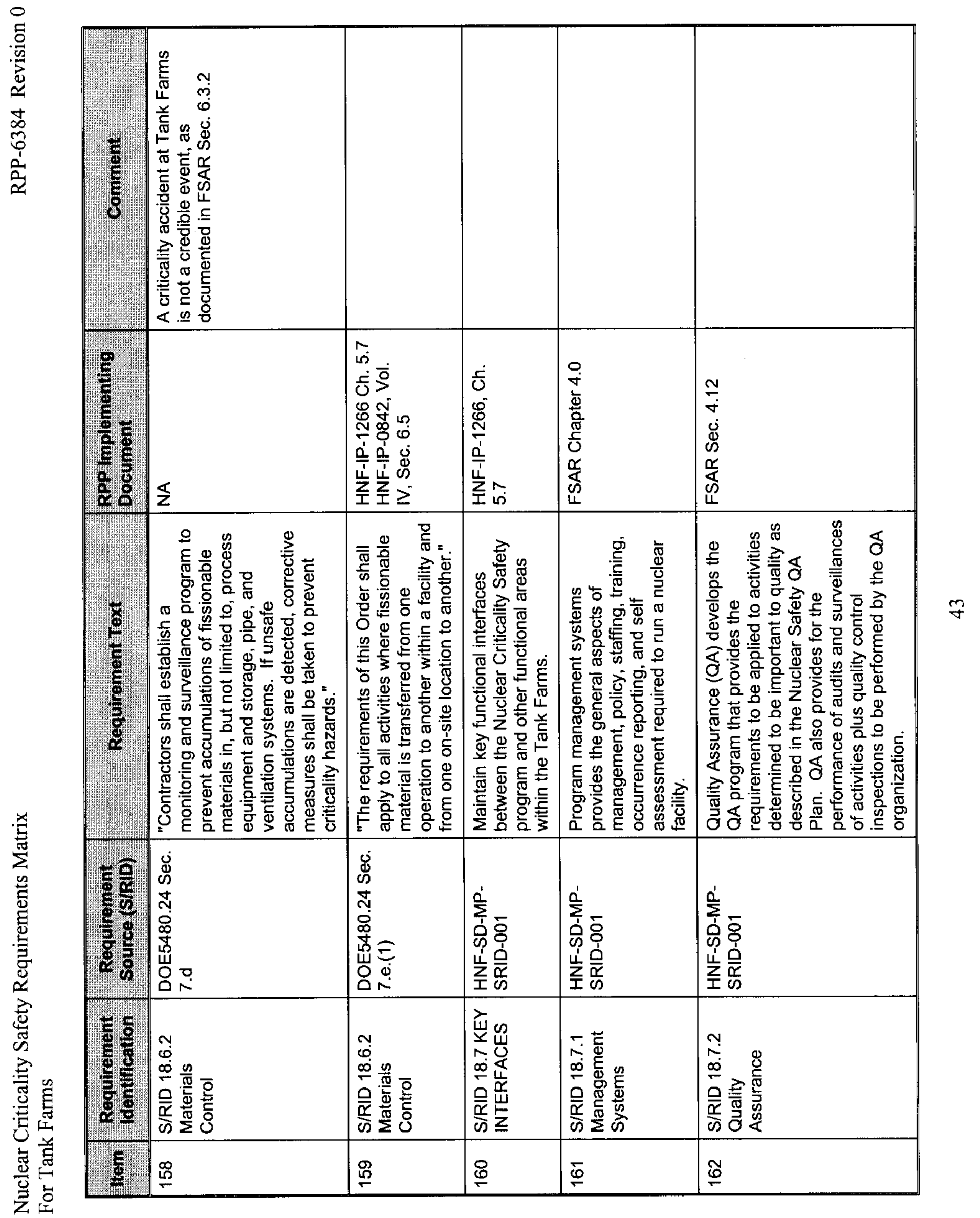




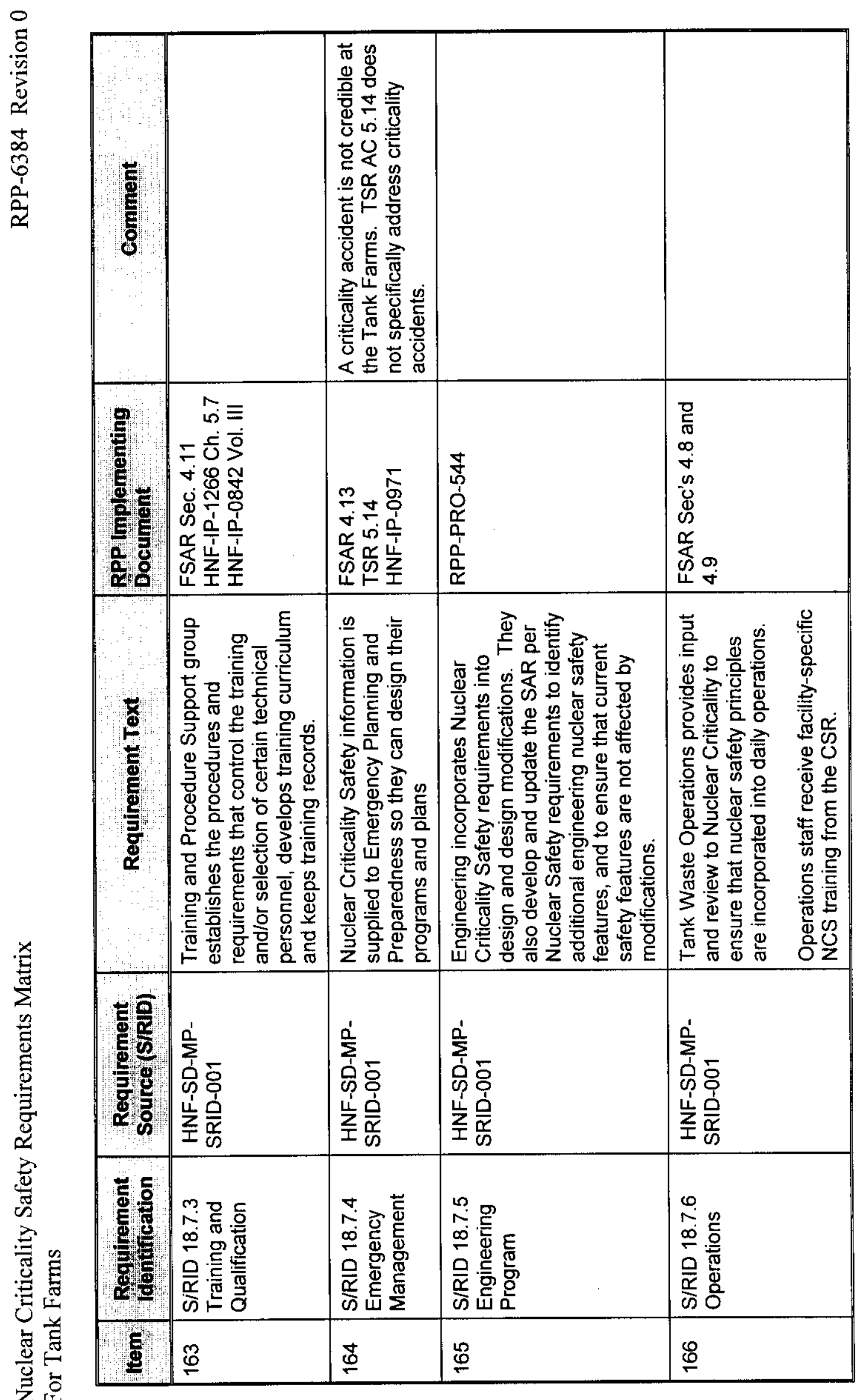




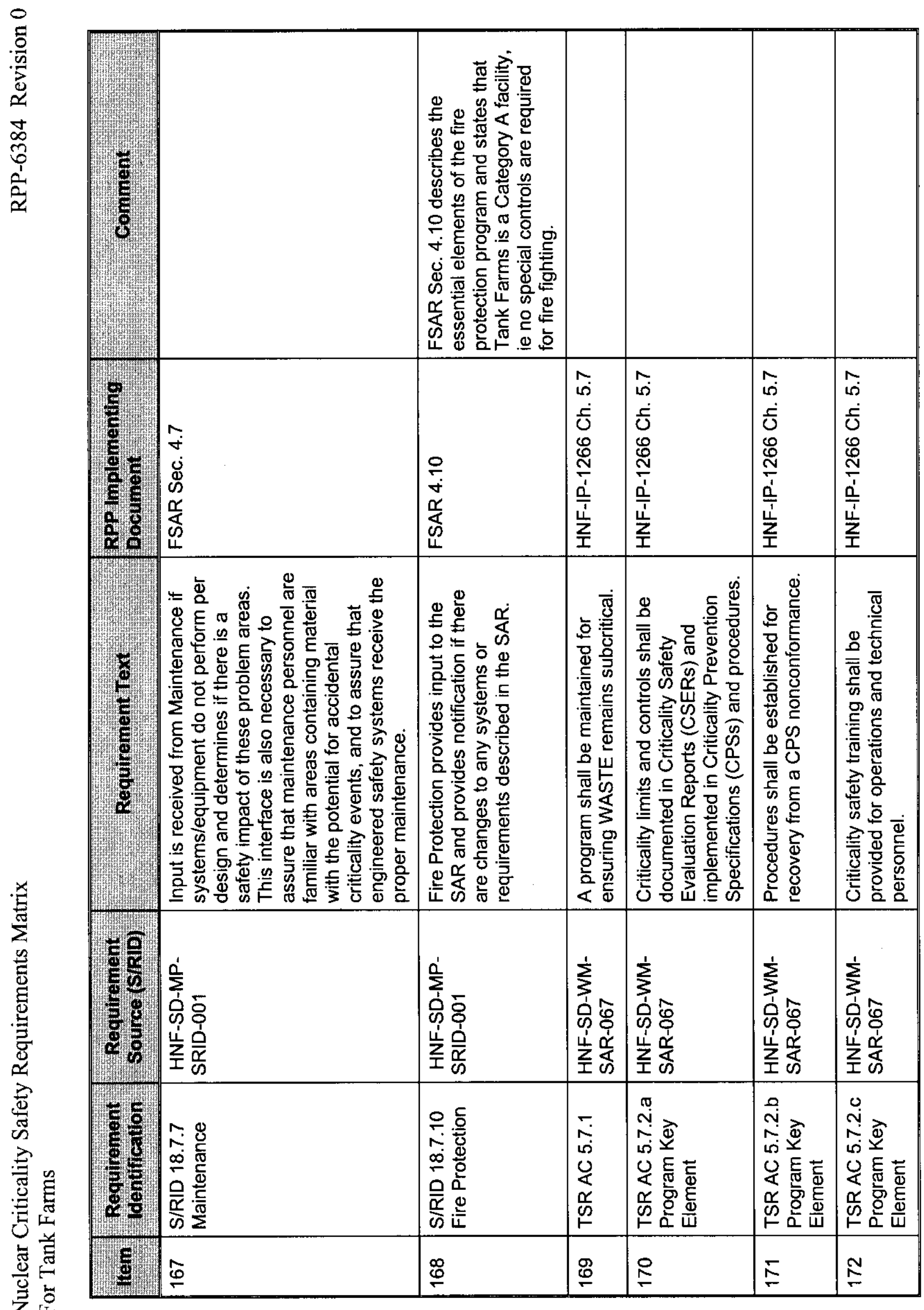




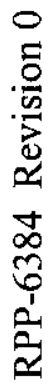

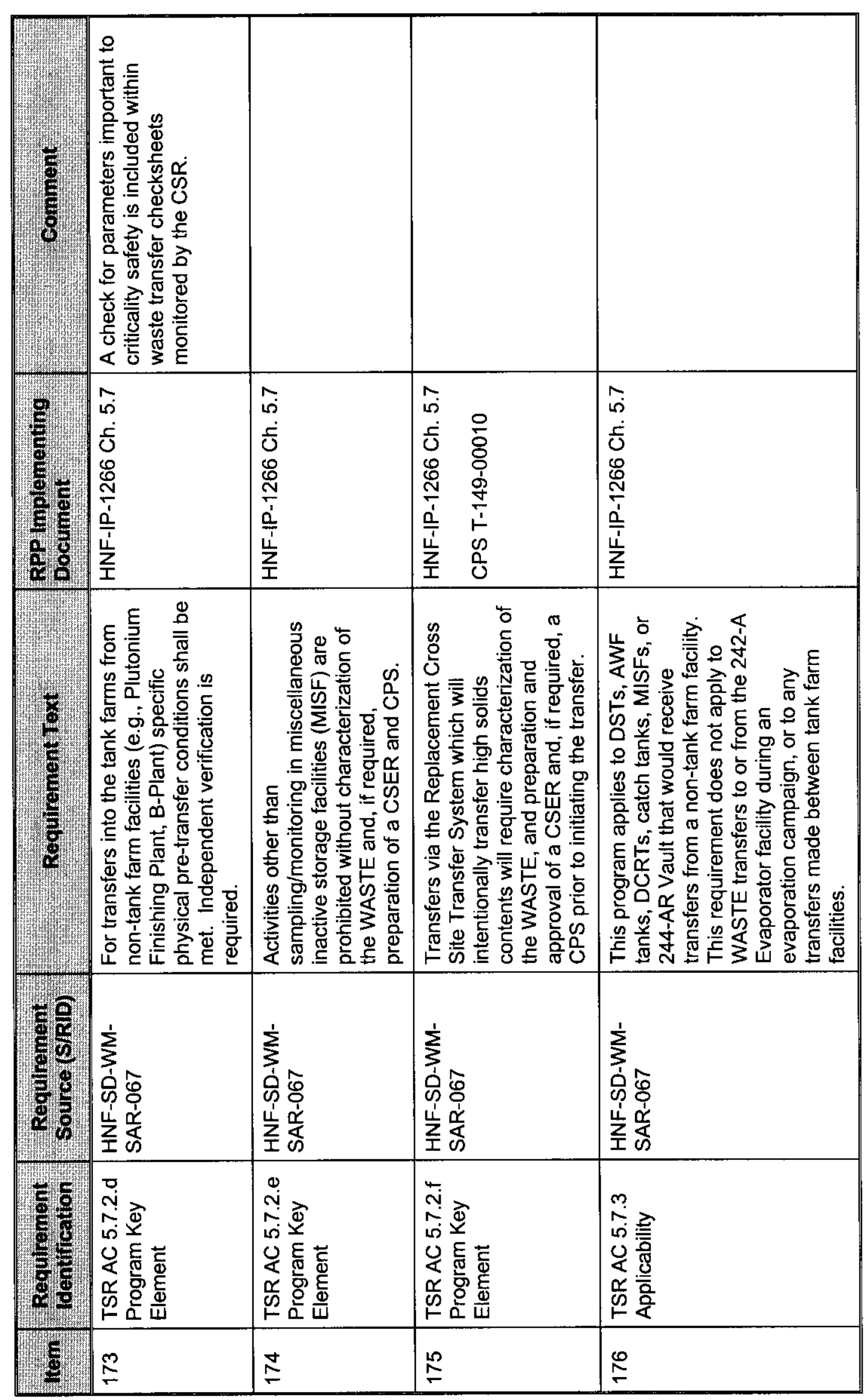




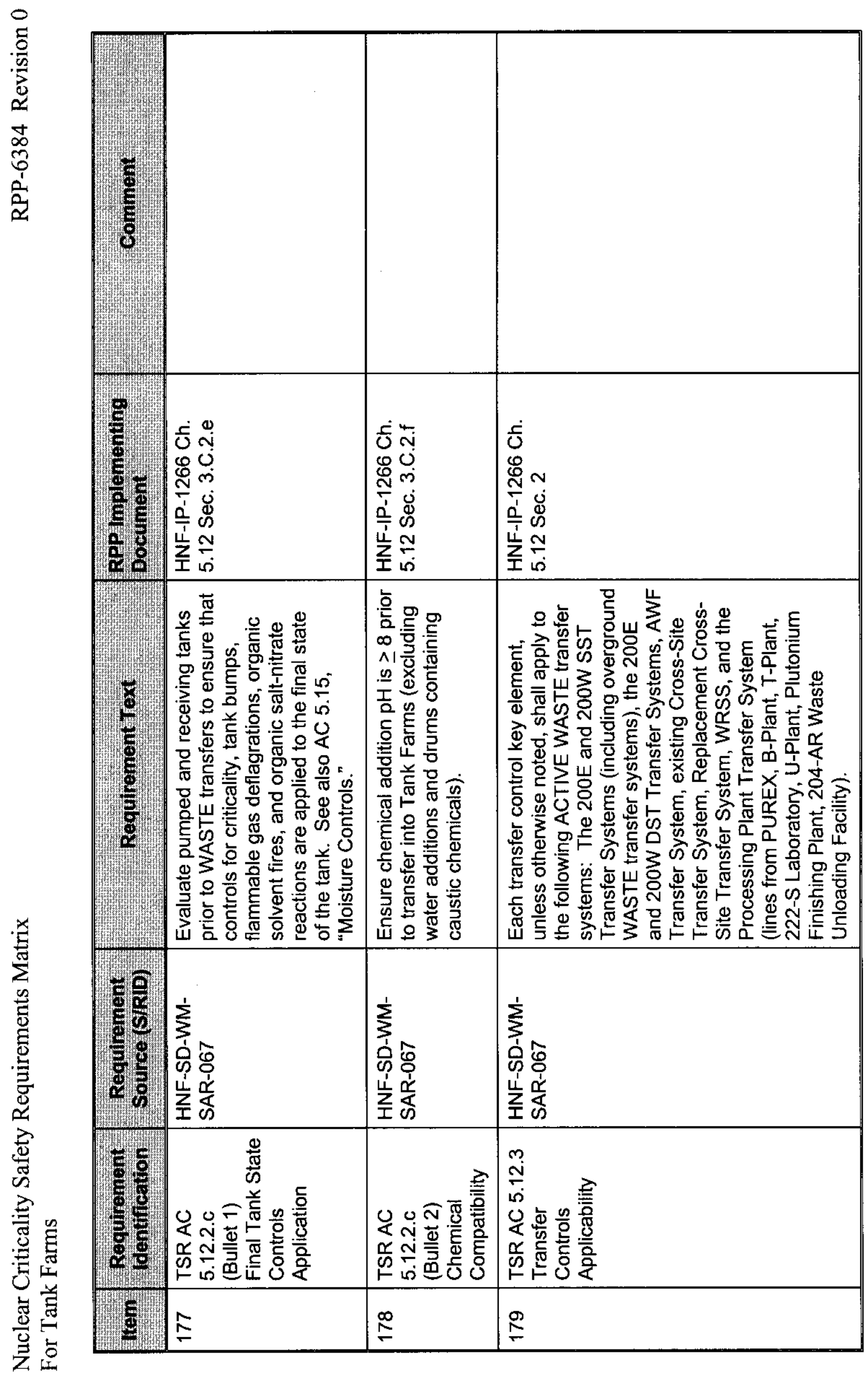

$\frac{1}{\grave{c}}$ 


\subsection{REFERENCES}

DOE O 420.1, Facility Safety, 1996, Change 2, Department of Energy, Washington, D.C..

DOE 5480.24, Nuclear Criticality Safety, 1992, Department of Energy, Washington, D.C.

HNF-IP-1266, Tank Farms Operations Administrative Controls, current version as of May 10, 2000, CH2MHill Hanford Group, Incorporated, Richland, Washington.

HNF-SD-MP-SRID-001, 1999, High Level Waste Storage Tank Farms/242-A Evaporator Standards/Requirements Identification Document, Rev. 2, CH2MHill Hanford Group, Incorporated, Richland, Washington.

HNF-SD-WM-SAR-067, 2000, Tank Waste Remediation System Final Safety Analysis Report, Rev. 1H, CH2MHill Hanford Group, Incorporated, Richland, Washington.

HNF-SD-WM-TSR-006, 2000, Tank Waste Remediation System Technical Safety Requirements, Rev. 1H, CH2MHill Hanford Group, Incorporated, Richland, Washington.

Internal Memo, E. J. Lipke to T. C. Geer, "Criticality Compliance," September 3, 1997, Attachment 2 " 9454500, Tank Farms Compliance Assessment and Implementation Plan for DOE Order 5480.24 Nuclear Criticality Safety, ” DE\&S Hanford, Richland, Washington.

TWR-3721, M. C. Brady Rapp et al, Tank Waste Remediation System Nuclear Criticality Safety Program Management Review, April 1999, DE\&S Hanford Corporation, Richland, Washington.

WHC-SD-WM-TI-725, 1996, Tank Farm Nuclear Criticality Review, Rev. 0A, Westinghouse Hanford Company, Richland, Washington. 


\section{DISTRIBUTION SHEET}

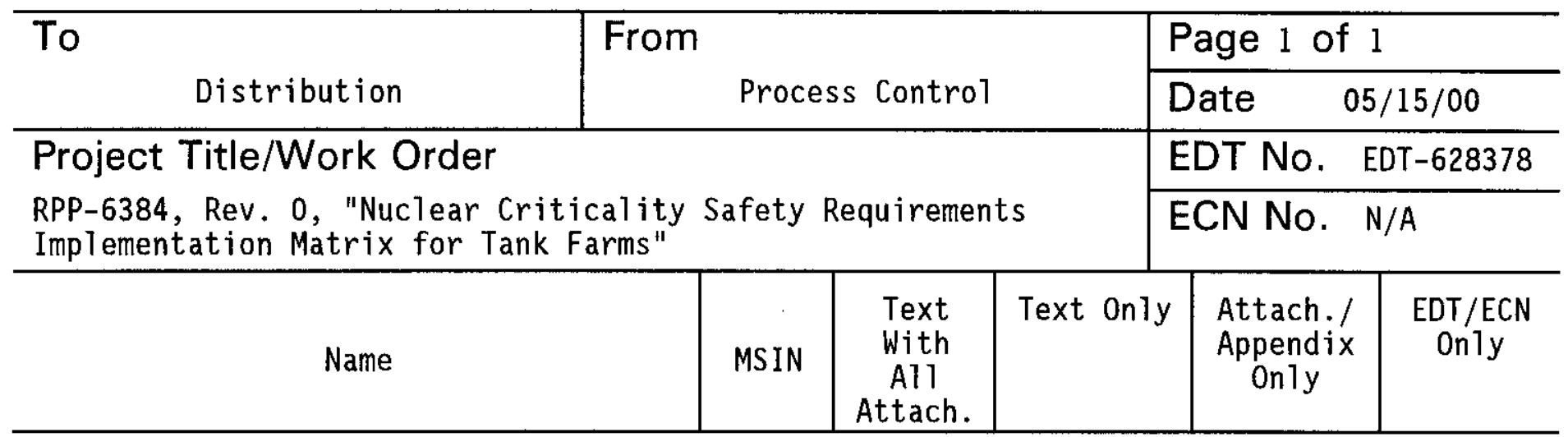

CH2M Hill Hanford Group, Inc.

M. J. Brown

C. DeFigh-Price

S6-15 $\quad X$

R. A. Dodd

L. A. Fort

K. D. Fowler

C. J. Hopkins

W. J. Kennedy

$\mathrm{R} 2-12 \quad 3$

R3-72 $\quad X$

R2-12 $\quad X$

R2-11 X

R2-50 $X$

N. W. Kirch

R. G. Stickney

L. E. Thomas

E. V. Weiss

T.C.S.R.C.

S5-07

R2-11

$\mathrm{R} 1-43$

S7-86

$\mathrm{R} 2-12$

$\mathrm{R} 1-10$

$X$
$X$
$X$
$X$
$X$
$X$

Eluor Hanford

G. R. Franz

A0-26 $\mathrm{X}$

E. J. Lipke

AO- 26

T5-54

B. S. Mo

T4-56

T. S. Vai1

\section{Fluor Federal Services}

H. Toffer

B4-44

$x$

Lockheed Mart in Services, Inc.

\section{Central Files}

Office of River Protection

J. S. Shuen

J. D. Voice

DOE Reading Room

Numatec Hanford Corporation

A. F. Choho
B1-07 X

H6-60 2

H6-60 X

$\mathrm{H} 2-53 \quad \mathrm{X}$

R3-73 X

Waste Management Federal Services of Hanford, Inc.

R. J. Nicklas

T4-56 $X$

